TRANSACTIONS OF THE

AMERICAN MATHEMATICAL SOCIETY

Volume 351, Number 2, February 1999, Pages 715-734

S $0002-9947(99) 02362-4$

\title{
GALOIS COVERINGS OF SELFINJECTIVE ALGEBRAS BY REPETITIVE ALGEBRAS
}

\author{
ANDRZEJ SKOWROŃSKI AND KUNIO YAMAGATA
}

\begin{abstract}
In the representation theory of selfinjective artin algebras an important role is played by selfinjective algebras of the form $\widehat{B} / G$ where $\widehat{B}$ is the repetitive algebra of an artin algebra $B$ and $G$ is an admissible group of automorphisms of $\widehat{B}$. If $B$ is of finite global dimension, then the stable module category $\bmod \widehat{B}$ of finitely generated $\widehat{B}$-modules is equivalent to the derived category $D^{b}(\bmod B)$ of bounded complexes of finitely generated $B$-modules. For a selfinjective artin algebra $A$, an ideal $I$ and $B=A / I$, we establish a criterion for $A$ to admit a Galois covering $F: \widehat{B} \rightarrow \widehat{B} / G=A$ with an infinite cyclic Galois group $G$. As an application we prove that all selfinjective artin algebras $A$ whose Auslander-Reiten quiver $\Gamma_{A}$ has a non-periodic generalized standard translation subquiver closed under successors in $\Gamma_{A}$ are socle equivalent to the algebras $\widehat{B} / G$, where $B$ is a representation-infinite tilted algebra and $G$ is an infinite cyclic group of automorphisms of $\widehat{B}$.
\end{abstract}

\section{INTRODUCTION}

In the paper, by an algebra is meant a basic, connected, artin algebra (associative, with an identity) over a commutative artin ring $K$. For an algebra $\Lambda$ we denote by $\bmod \Lambda$ the category of finitely generated right $\Lambda$-modules and by $D: \bmod \Lambda \rightarrow \bmod \Lambda^{\text {op }}$ the standard duality $\operatorname{Hom}_{K}(-, E)$ where $E$ is a minimal injective cogenerator in $\bmod K$. An algebra $\Lambda$ is called selfinjective if $\Lambda \simeq D(\Lambda)$ in $\bmod \Lambda$, that is, the projective $\Lambda$-modules are injective. If $\Lambda$ is selfinjective, then the left and the right socle of $\Lambda$ coincide, and we denote them by $\operatorname{soc} \Lambda$. Two selfinjective algebras $\Lambda$ and $R$ are said to be socle equivalent if the factor algebras $\Lambda / \operatorname{soc} \Lambda$ and $R / \operatorname{soc} R$ are isomorphic. Frequently, selfinjective algebras are socle equivalent to selfinjective algebras having triangular Galois coverings, and then we may reduce the study of such algebras and their representations to that for the corresponding algebras of finite global dimension. This is the case for all representation-finite selfinjective algebras over algebraically closed fields [6], [19] and certain classes of tame representation-infinite selfinjective algebras [1], [2], [9], [11], [22]. An important class of selfinjective algebras is formed by the algebras of the form $\widehat{B} / G$ where

Received by the editors February 4, 1997.

1991 Mathematics Subject Classification. Primary 16D50, 16G10, 16G70, 16S99.

(C)1999 American Mathematical Society 
$\widehat{B}$ is the repetitive algebra [15] (locally bounded, without identity)

$$
\widehat{B}=\left[\begin{array}{cccccc}
\ddots & \ddots & & & & \\
& B_{m-1} & Q_{m-1} & & & \\
& & & & & \\
& & B_{m} & Q_{m} & & \\
& & & B_{m+1} & Q_{m+1} & \\
& & & & \ddots & \ddots
\end{array}\right]
$$

of an algebra $B$, where $B_{m}=B$ and $Q_{m}={ }_{B} D(B)_{B}$ for all $m \in \mathbb{Z}$, all the remaining entries are zero, the matrices in $\widehat{B}$ have only finitely many nonzero elements, addition is the usual addition of matrices, and multiplication is induced from the canonical maps $B \otimes_{B} D(B) \rightarrow D(B), D(B) \otimes_{B} B \rightarrow D(B)$, and the zero map $D(B) \otimes_{B} D(B) \rightarrow 0$, and $G$ is an admissible group of $K$-automorphisms of $\widehat{B}$. We note that if $B$ is of finite global dimension, then the stable module category $\bmod \widehat{B}$ of $\bmod \widehat{B}$ is equivalent, as a triangulated category, to the derived category $D^{b}$ $(\bmod B)$ of bounded complexes over $\bmod B[13]$.

In the paper we are interested in the question of when a selfinjective algebra $A$ is isomorphic (respectively, socle equivalent) to an algebra of the form $\widehat{B} / G$, where $B$ is a factor algebra $A / I$ of $A$ by an ideal $I$, and $G$ is an admissible infinite cyclic group of automorphisms of $\widehat{B}$. Our main results give sufficient conditions for a selfinjective algebra $A$ to be of the above form. Moreover, we determine the structure of certain classes of selfinjective algebras whose Auslander-Reiten quiver has a prescribed form.

We shall now sketch the content of the paper. In Section 2 we fix notations and prove some preliminary results applied in the proofs of our main results. The main result proved in Section 3 is the following theorem. Let $A$ be a basic and connected selfinjective artin algebra, $I$ a (two-sided) ideal of $A, B=A / I$, and $e$ a residual identity of $B$, that is, a minimal idempotent of $A$ representing the identity of $B$. Denote by $\nu$ the Nakayama automorphism of $A$, and by $\widetilde{e}$ the sum of idempotents forming the $\nu$-orbits of pairwise nonisomorphic primitive summands of the idempotent $e$. Assume that: (1) $\widetilde{e}(I e I) \widetilde{e}=0 ;(2) \nu^{-}(e) I e$ and $e I \nu(e)$ are injective cogenerators as a right $e A e / e I e$-module and a left $e A e / e I e$-module, respectively, and (3) the canonical algebra epimorphism $e A e \rightarrow e A e / e I e$ splits. Then $\widetilde{e} A \widetilde{e} \simeq \widehat{B} / G$ for an admissible infinite cyclic group $G$ of automorphisms of $\widehat{B}$. We also note that $\widetilde{e}=1$ if $I e I=0$.

In the final two sections we derive some consequences of the above theorem and the main results proved in our paper [26]. In Section 4 we prove that if $A$ is a basic, connected, selfinjective artin algebra, $I$ an ideal of $A, B=A / I$, e a residual identity of $B$, and such that $I e I=0, I e$ is an injective cogenerator in $\bmod B$, and the ordinary quiver $Q_{B}$ of $B$ has no oriented cycles, then $A$ is socle equivalent to $\widehat{B} / G$ for some admissible infinite cyclic group $G$ of automorphisms of $\widehat{B}$. Moreover, $A \simeq \widehat{B} / G$ if $K$ is an algebraically closed field. In Section 5, we prove that if $A$ is a basic and connected selfinjective artin algebra whose Auslander-Reiten 
quiver $\Gamma_{A}$ contains a non-periodic generalized standard right stable full translation subquiver which is closed under successors in $\Gamma_{A}$, then $A$ is socle equivalent to an algebra of the form $\widehat{B} / G$, where $B$ is a representation-infinite tilted algebra and $G$ is an admissible infinite cyclic group of automorphisms of $\widehat{B}$. Moreover, if $K$ is an algebraically closed field, then $A$ is isomorphic to $\widehat{B} / G$.

For basic background concerning the representation theory applied here we refer to $[3],[20],[23],[29]$.

The results of the paper were partially announced by the authors during the conferences at Cocoyoc (August 1994) and Padova (June 1994). The research was initiated during the stay of the second named author in Torun and completed when the first named author visited Tsukuba. Both authors gratefully acknowledge the hospitality of each other's university and support from the Polish Scientific Grant KBN No. 2P03A 02008 and the Grant in Aid for Scientific Research (C) No. 08640008, the Ministry of Education of Japan.

\section{Preliminary Results}

Throughout the paper, $A$ will denote a fixed basic and connected selfinjective artin algebra over a commutative artin ring $K$, and $\left\{e_{i} \mid 1 \leq i \leq s\right\}$ a (complete) set of primitive orthogonal idempotents of $A$ such that $1=e_{1}+\ldots+e_{s}$.

Let $\sigma$ be an algebra automorphism of $A$. For a right $A$-module $M, M_{\sigma}$ denotes the right $A$-module obtained from $M$ by changing the operation of $A$ as follows: $m \cdot a=m \sigma(a)$ for each $a \in A$ and $m \in M$. Similarly, for a left $A$-module $N$, ${ }_{\sigma} N$ denotes the left $A$-module obtained from $N$ by changing the operation of $A$ as follows: $a \cdot m=\sigma(a) m$ for each $a \in A$ and $m \in M$.

Let $\nu$ be the Nakayama automorphism of $A$ with an $A$-bimodule isomorphism $\Theta_{r}: A \rightarrow{ }_{\nu} D(A)$. Hence we have $\operatorname{soc}\left(e_{i} A\right) \simeq \operatorname{top}\left(\nu\left(e_{i}\right) A\right)\left(=\nu\left(e_{i}\right) A / \nu\left(e_{i}\right)(\operatorname{rad} A)\right)$ as right $A$-modules for all $i \in\{1, \ldots, s\}$. Since $\left\{\nu\left(e_{i}\right) A \mid 1 \leq i \leq s\right\}$ is a set of representatives of indecomposable projective right $A$-modules, there is a permutation of $\{1, \ldots, n\}$, denoted again by $\nu$, such that $\nu\left(e_{i}\right) A \simeq e_{\nu\left(e_{i}\right)} A$ for all $i \in\{1, \ldots, s\}$. Invoking the Krull-Schmidt theorem we may assume that $\nu\left(e_{i} A\right)=\nu\left(e_{i}\right) A=e_{\nu(i)} A$ for all $i \in\{1, \ldots, s\}$. Consider now the $K$-linear isomorphism ()$^{* *}: A \rightarrow D(D(A))$ given by $a^{* *}(f)=f(a)$ for each $a \in A$ and $f \in D(A)$. Then a direct checking shows that $\Theta_{l}=D\left(\Theta_{r}\right)()^{* *}: A \rightarrow D(A)_{\nu^{-}}$is an $A$-bimodule isomorphism. Moreover, we have the following fact.

Lemma 2.1. The Nakayama automorphism $\nu$ coincides with the composite $\Theta_{l}^{-1} \Theta_{r}: A \rightarrow{ }_{\nu} A_{\nu}$ as an A-bimodule isomorphism.

Proof. First observe that $\Theta_{l}\left(1_{A}\right)=D\left(\Theta_{r}\right)\left(1_{A}^{* *}\right)=1_{A}^{* *} \Theta_{r}$. Then, for each $a \in A$, we get $\Theta_{l}\left(1_{A}\right)(a)=1^{* *} \Theta_{r}(a)=1^{* *}\left(\Theta_{r}\left(1_{A}\right) a\right)=\left(\Theta_{r}\left(1_{A}\right) a\right)\left(1_{A}\right)=\Theta_{r}\left(1_{A}\right)\left(a \cdot 1_{A}\right)=$ $\Theta_{r}\left(1_{A}\right)(a)$, and hence $\Theta_{l}\left(1_{A}\right)=\Theta_{r}\left(1_{A}\right)$. Further, $\left(\Theta_{l}^{-1} \Theta_{r}\right)(a)=\left(\Theta_{l}^{-1} \Theta_{r}\right)\left(a 1_{A}\right)=$ $\Theta_{l}^{-1} \Theta_{r}\left(a 1_{A}\right)=\Theta_{l}^{-1}\left(\nu(a) \Theta_{r}\left(1_{A}\right)\right)=\nu(a) \Theta_{l}^{-1} \Theta_{r}\left(1_{A}\right)=\nu(a)$, for any $a \in A$. This implies that $\nu=\Theta_{l}^{-1} \Theta_{r}$.

From now on we assume that $I$ is a (two-sided) ideal of $A, B=A / I$ and $e$ is an idempotent of $A$ such that $e+I$ is an identity of $B$. We may assume that $e=e_{1}+\ldots+e_{t}$ for some $t \leq s$, and $\left\{e_{i} \mid 1 \leq i \leq t\right\}$ is the subset of $\left\{e_{i} \mid 1 \leq i \leq s\right\}$ consisting of all idempotents $e_{i}$ which are not in $I$. Then such an idempotent $e$ is uniquely determined by $I$ up to an inner automorphism of $A$, and we call it a residual identity of $B$. Note that $B \simeq e A e / e I e$ and $1-e \in I$. An idempotent $e^{\prime}$ 
is called a summand of an idempotent $f$, and we write $e^{\prime}<f$, if $e^{\prime} f=f e^{\prime}=e^{\prime}$, and is said to be primitive if the right ideal $e^{\prime} A$ generated by $e^{\prime}$ is indecomposable. The residue class $e^{\prime}+I$ of $e^{\prime}$ in $B=A / I$ will also be denoted by $e^{\prime}$. The $\nu$-orbit idempotent of $e$, denoted by $\widetilde{e}$, is the sum of all $e_{i}$ of the form $e_{i}=e_{\nu^{m}(j)}$, for some $1 \leq j \leq t$ and an integer $m$.

Lemma 2.2. The algebra $\widetilde{e} A \widetilde{e}$ is selfinjective.

Proof. From definition of $\widetilde{e}$ we know that, for each idempotent $e_{i}$ with $e_{i}<\widetilde{e}$, $\operatorname{soc}\left(e_{i} A\right)=\operatorname{soc}\left(e_{i} A\right) \widetilde{e}$ is a simple socle of $e_{i} A \widetilde{e}$. Moreover, the socle of $\widetilde{e} A \widetilde{e}$, as a right $\widetilde{e} A \widetilde{e}-$ module, is a direct sum of pairwise nonisomorphic simple modules. Then the top of the left $\widetilde{e} A \widetilde{e}$-module $D(\widetilde{e} A \widetilde{e})$ is a direct sum of pairwise nonisomorphic simple modules, and consequently we have an epimorphism $\widetilde{e} A \widetilde{e} \rightarrow D(\widetilde{e} A \widetilde{e})$ of left $\widetilde{e} A \widetilde{e}$-modules. Since $\widetilde{e} A \widetilde{e}$ and $D(\widetilde{e} A \widetilde{e})$ have the same length as $K$-modules, we get $\widetilde{e} A \widetilde{e} \simeq D(\widetilde{e} A \widetilde{e})$ as left $\widetilde{e} A \widetilde{e}$-modules, and then also as right $\widetilde{e} A \widetilde{e}$-modules. Therefore $\widetilde{e} A \widetilde{e}$ is selfinjective.

The next lemma shows when $\widetilde{e}=1$.

Lemma 2.3. Assume $\widetilde{e}_{j} A e A \widetilde{e}_{j}=0$ for any $e_{j}<1-\widetilde{e}$. Then $\widetilde{e}=1$.

Proof. Suppose $\widetilde{e} \neq 1$. Since $A$ is connected, there exist $j \in\{1, \ldots, s\}$ and some integer $m$ such that $e_{j} A e_{\nu^{-m}(i)} \neq 0$ for some $e_{i}<e$. Applying $\nu^{m}$ we get $e_{\nu^{m}(j)} A e_{i} \neq$ 0 . But $\operatorname{soc}\left(e_{\nu^{m}(j)} A e_{i} A\right) \simeq \operatorname{top} e_{\nu^{m+1}(j)} A$. Thus $e_{\nu^{m}(j)} A e_{i} A e_{\nu^{m+1}(j)} \neq 0$, and hence $\widetilde{e}_{j} A e_{i} A \widetilde{e}_{j} \neq 0$. Since $e_{i}<e$ and $e_{j}<1-\widetilde{e}$, this contradicts our assumption.

Corollary 2.4. Assume $I e I=0$. Then $\widetilde{e}=1$.

Proof. Clearly $1-\widetilde{e} \in I$. Then $I e I=0$ implies $(1-\widetilde{e}) A e A(1-\widetilde{e})=0$, and consequently $\widetilde{e}=1$ by Lemma 2.3 .

The following lemma will be useful in our investigations.

Lemma 2.5. The following conditions are equivalent:

(i) $\widetilde{e}(I e I)=0$.

(ii) $($ IeI $) \widetilde{e}=0$.

(iii) $\widetilde{e}(I e I) \widetilde{e}=0$.

(iv) $\tilde{e} I e=\nu^{-}(e) I e$ and $\nu^{-}(e) I e I e=0$.

(v) $\tilde{e} I e=\nu^{-}(e) I e$ and $\nu^{-}(e) I e I=0$.

Proof. The implications (ii) $\Rightarrow$ (iii) and (v) $\Rightarrow$ (i) are obvious. For (i) $\Rightarrow($ ii), suppose that $I e I \widetilde{e} \neq 0$. Then $\widetilde{e}(I e I) \widetilde{e} \neq 0$, as a left $A$-module, because $\widetilde{e}\left(\operatorname{soc}_{A}(I e I \widetilde{e})\right) \neq 0$. Hence $\widetilde{e}(I e I) \neq 0$. For (iv) $\Rightarrow(\mathrm{v})$ observe that if $\nu^{-}(e) I e I \neq 0$, then $\left(\nu^{-}(e) I e I\right) e \neq$ 0 . Therefore, it remains to show that (iii) implies (iv). Assume $\widetilde{e}(I e I) \widetilde{e}=0$. Since $e<\widetilde{e}$ and $\nu^{-}(e)<\widetilde{e}$ we then get $\nu^{-}(e) I e I e=\nu^{-}(e)(I e I) e=0$. In order to prove the first claim it suffices to show that $\left(\widetilde{e}-\nu^{-}(e)\right) I e=0$. Suppose that $\left(\widetilde{e}-\nu^{-}(e)\right) I e \neq 0$. Then $\left(\widetilde{e}-\nu^{-}(e)\right) \operatorname{IeA}(\widetilde{e}-e) \neq 0$ because $\nu\left(\widetilde{e}-\nu^{-}(e)\right)=$ $\widetilde{e}-e$ and $\operatorname{soc}\left(\left(\widetilde{e}-\nu^{-}(e)\right) I e A\right)(\widetilde{e}-e) \neq 0$. Since $\widetilde{e}-e \in I$, this implies that $\left(\widetilde{e}-\nu^{-}(e)\right) \operatorname{IeI}(\widetilde{e}-e) \neq 0$, which contradicts our assumption $\widetilde{e}(I e I) \widetilde{e}=0$. This finishes the proof.

For a subset $S$ of $A$, let $l_{S}(I)$ be the left annihilator $\{a \in S \mid a I=0\}$ of $I$ in $S$ and $r_{S}(I)$ the right annihilator $\{a \in S \mid I a=0\}$ of $I$ in $S$. Then $r_{A} l_{A}(I)=I$ and $l_{A} r_{A}(I)=I$, and in particular we have $l_{A}(I) \neq 0$ and $r_{A}(I) \neq 0$. The 
following lemma proved in [26, Lemma 1.1] shows that a minimal cogenerator in $\bmod B$, or in $\bmod B^{\text {op }}$, can be obtained as an ideal in $A$.

Lemma 2.6. The right ideal $\nu^{-}(e) l_{A}(I)$ is a minimal injective cogenerator in $\bmod B$, and the left ideal $r_{A}(I) \nu(e)$ is a minimal injective cogenerator in mod $B^{\mathrm{op}}$.

In the next three lemmas we assume that the ideal $I$ satisfies the following two conditions:

(1) $\widetilde{e}(I e I) \widetilde{e}=0$.

(2) $\nu^{-}(e) I e$ is an injective cogenerator in $\bmod e A e / e I e$ and $e I \nu(e)$ is an injective cogenerator in $\bmod (e A e / e I e)^{\mathrm{op}}$.

Lemma 2.7. For each integer $m, \nu^{m}\left(\nu^{-}(e) I e\right)$ and $\nu^{m}(e I \nu(e))$ have A-bimodule structures, and there are isomorphisms

$$
\begin{aligned}
\Theta_{r}^{\prime}: \nu^{-}(e) I e & \rightarrow{ }_{\nu} D(B), & \Theta_{l}^{\prime}: e I \nu(e) & \rightarrow D(B)_{\nu^{-}}, \\
\Theta_{r}^{(m)}: \nu^{m}\left(\nu^{-}(e) I e\right) & \rightarrow{ }_{\nu} D\left(\nu^{m}(B)\right), & \Theta_{l}^{(m)}: \nu^{m}(e I \nu(e)) & \rightarrow D\left(\nu^{m}(B)\right)_{\nu^{-}}
\end{aligned}
$$

of A-bimodules such that, for the canonical epimorphism $\rho: A \rightarrow A / I=B$, the following diagrams are commutative:

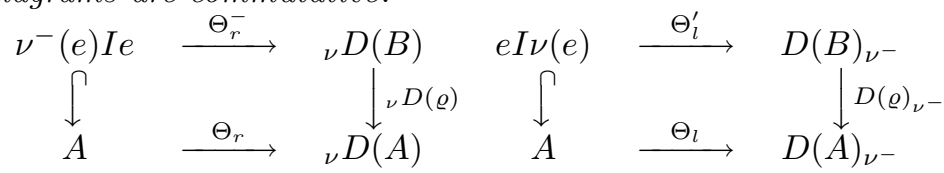

where $\nu^{m}(B)$ denotes the factor algebra $A / \nu^{m}(I)$ and $\nu^{m}(b)=\nu^{m}(a)+\nu^{m}(I)$ for $b=a+I \in B$.

Proof. Clearly $B$ is an $A$-bimodule and the canonical epimorphism $\varrho: A \rightarrow B$ is an $A$-bimodule epimorphism. Hence $D(\varrho): D(B) \rightarrow D(A)$ is an $A$-bimodule monomorphism. Let $\varphi=\Theta_{r}^{-1}{ }_{\nu} D(\varrho):{ }_{\nu} D(B) \rightarrow{ }_{\nu} D(A) \rightarrow A$. Then ${ }_{\nu} D(B) \simeq$ $\varphi\left({ }_{\nu} D(B)\right)$ as $A$-bimodules. Since $D(B) I=0$ and $D(B)=e D(B) e$, we conclude that $\varphi\left({ }_{\nu} D(B)\right)$ is a $B$-submodule of $\nu^{-}(e) l_{A}(I)_{B}$. Further, it folows from Lemma 2.6 that $\nu^{-}(e) l_{A}(I)_{B}$ is isomorphic to $D(B)_{B}$, and consequently we get $\varphi\left({ }_{\nu} D(B)\right)=\nu^{-}(e) l_{A}(I)_{B}$. Moreover, by our assumption (1) and Lemma 2.5, we have $\nu^{-}(e) I e \subseteq l_{\nu^{-}(e) A e}(I)$. On the other hand, by our assumption $(2),\left(\nu^{-}(e) I e\right)_{B}$ is an injective cogenerator in $\bmod B$. This implies $\varphi\left({ }_{\nu} D(B)\right)=\nu^{-}(e) I e$, because $1-e \in I$ and $l_{\nu^{-}(e) A e}(I)=\nu^{-}(e) l_{A}(I)$. In particular, $\nu^{-}(e) I e$ is an $A$-bimodule and $\varphi$ is an $A$-bimodule isomorphism. Hence $\Theta_{r}^{\prime}=\varphi^{-1}: \nu^{-}(e) I e \rightarrow{ }_{\nu} D(B)$ satisfies the required conditions. Dually, we prove that there exists $\Theta_{l}^{\prime}: e I \nu(e) \rightarrow D(B)_{\nu^{-}}$ satisfying the required conditions. The isomorphisms $\Theta_{r}^{(m)}$ and $\Theta_{l}^{(m)}$ are then easily obtained by substituting $\nu^{m}(I)$ for $I$ and $\nu^{m}(B)$ for $B$ in the above argument, because $\nu^{m}: A \rightarrow A$ is an algebra isomorphism.

Lemma 2.8. For any $1 \leq i \leq t$ and any integer $m$, the following inclusions hold:

$$
\nu^{m-1}\left(e_{i}\right) I e \subseteq \nu^{-}(e) I e \quad \text { and } e I \nu^{m}\left(e_{i}\right) \subseteq e I \nu(e) .
$$

Moreover, the restrictions of $\Theta_{r}^{\prime}$ and $\Theta_{l}^{\prime}$ induce the following isomorphisms:

$$
\nu^{m-1}\left(e_{i}\right) I e \simeq \nu^{m}\left(e_{i}\right) D(B) e \quad \text { and } \quad e I \nu^{m}\left(e_{i}\right) \simeq e D(B) \nu^{m-1}\left(e_{i}\right) .
$$

Proof. Fix $1 \leq i \leq t$ and an integer $m$. Assume first that $\nu^{m-1}\left(e_{i}\right)<\nu^{-}(e)$. Then $\nu^{m-1}\left(e_{i}\right) \nu^{-}(e)=\nu^{m-1}\left(e_{i}\right)$ and it follows from Lemma 2.7 that $\Theta_{r}^{\prime}$ induces an isomorphism $\nu^{m-1}\left(e_{i}\right) I e \simeq \nu^{m}\left(e_{i}\right) D(B) e$. Assume now $\nu^{m-1}\left(e_{i}\right) \nless \nu^{-}(e)$. In 
this case, $\nu^{m-1}\left(e_{i}\right)<\widetilde{e}-\nu^{-}(e)$. Applying now Lemma 2.5 and our assumption (1) we get $\nu^{m-1}\left(e_{i}\right) I e=0$. On the other hand, $\nu^{m}\left(e_{i}\right)<\nu(\widetilde{e})-\nu\left(\nu^{-}(e)\right)=$ $\widetilde{e}-e$, and so we have $\nu^{m}\left(e_{i}\right) D(B) e=0$. Thus $\Theta_{r}^{\prime}$ induces the trivial isomorphism $\nu^{m-1}\left(e_{i}\right) I e \simeq \nu^{m}\left(e_{i}\right) D(B) e$ between the zero modules. The proof of the remaining part is dual.

Lemma 2.9. For any $1 \leq i, j \leq t$ and any integer $m$,

$$
e_{\nu^{m}(i)} \nu^{m}(I) e_{\nu^{m+1}(j)}=e_{\nu^{m}(i)} \nu^{m+1}(I) e_{\nu^{m+1}(j)},
$$

and the following diagram is commutative:

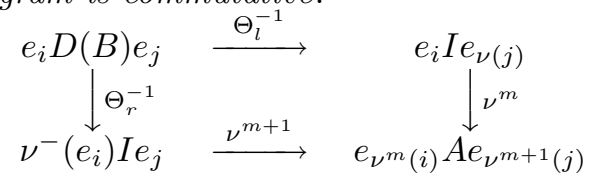

Proof. Let $1 \leq i, j \leq t$ and $m \in \mathbb{Z}$. We know from Lemma 2.1 that $\nu=\Theta_{l}^{-1} \Theta_{r}$. Moreover, by Lemma 2.7, $\Theta_{r}$ induces an isomorphism $\nu^{-}\left(e_{i}\right) I e_{j} \stackrel{\sim}{\longrightarrow} e_{i} D(B) e_{j}$ and $\Theta_{l}$ an isomorphism $e_{i} I e_{\nu(j)} \rightarrow e_{i} D(B) e_{j}$. Hence

$$
e_{i} I e_{\nu(j)}=\nu\left(\nu^{-}\left(e_{i}\right) I e_{j}\right)=e_{i} \nu(I) e_{\nu(j)} .
$$

Applying $\nu^{m}$ we then get

$$
e_{\nu^{m}(i)} \nu^{m}(I) e_{\nu^{m+1}(j)}=e_{\nu^{m}(i)} \nu^{m+1}(I) e_{\nu^{m+1}(j)} .
$$

\section{Galois COVERINGS of SELFinjeCtive AlgEBras}

Recall that a $K$-category $R$ is called locally bounded [5] if the following conditions are satisfied:

(a) distinct objects of $R$ are not isomorphic;

(b) the algebras $R(x, x)$ are local;

(c) for each object $x$ of $R, \sum_{y \in R}|R(x, y)|$ and $\sum_{y \in R}|R(y, x)|$ are finite.

Here, for a $K$-module $V$, we denote by $|V|$ its length over $K$. A functor $F: R \rightarrow \Lambda$ between two locally bounded $K$-categories $R$ and $\Lambda$ is called a covering functor if the induced maps

$$
\bigoplus_{F(y)=a} R(x, y) \rightarrow \Lambda(F(x), a) \quad \text { and } \quad \bigoplus_{F(y)=a} R(y, x) \rightarrow \Lambda(a, F(x))
$$

are isomorphisms for all objects $x \in R$ and $a \in \Lambda$ (see [5], [12]).

Let $R$ be a locally bounded $K$-category and $G$ a group of $K$-linear automorphisms of $R$. We assume that $G$ acts freely on the objects of $R$, that is, $g x \neq x$ for each object $x$ of $R$ and $g \neq 1$ in $G$. It follows from [12, Proposition 3.1] that the quotient $R / G$ exists in the category of locally bounded $K$-categories and there is a canonical covering functor $F: R \rightarrow R / G$. Moreover, $F$ is universal with respect to the property $F g=F$ for each $g \in G$, that is, each functor $E: R \rightarrow \Lambda$ which satisfies $E g=E$ for each $g \in G$ admits a unique factorization $E=H F$. The objects of $R / G$ are the orbits of $G$ in the set of objects of $R$. A morphism $f: a \rightarrow b$ between two objects in $R / G$ is a family $f=\left({ }_{y} f_{x}\right) \in \prod_{x, y} R(x, y)$, where $x, y$ range over $a, b$, respectively, and $f$ satisfies the relation $g\left({ }_{y} f_{x}\right)={ }_{g y} f_{g x}$ for all $g \in G$ and all $x, y$. The composition ef of $f: a \rightarrow b$ and $e: b \rightarrow c$ in $R / G$ is defined by ${ }_{z} e f_{x}=\sum_{y \in b} e_{y} f_{x}$ (this sum makes sense since $R$ is locally bounded). Then we 
have the canonical covering functor $F: R \rightarrow R / G$ which assigns to each object $x$ of $R$ its $G$-orbit, and to a morphism $\xi \in R(x, y)$ the family $F \xi=\left({ }_{h y} F \xi_{g x}\right)_{g, h \in G}$ such that ${ }_{h y} F \xi_{g x}=g \xi$ if $g=h$ and ${ }_{h y} F \xi_{g x}=0$ for $g \neq h$. Frequently, we are dealing with a $K$-linear functor $E: R \rightarrow \Lambda$ such that $E g=E$ for all $g \in G$. Such a functor induces an isomorphism $R / G \simeq \Lambda$ if and only if $E$ is surjective on the objects and $G$ acts transitively on the fiber $E^{-1}(a)$ of each object $a$ of $\Lambda$. If this is the case, the functor $E: R \rightarrow \Lambda$ is called a Galois covering. Clearly, the above-defined functor $F: R \rightarrow R / G$ is a Galois covering. We refer to [5], [12] for more details on covering functors.

We may consider the algebra $A$ as a (locally bounded) $K$-category whose set of objects is the fixed set $\left\{e_{i} \mid 1 \leq i \leq s\right\}$ of primitive orthogonal idempotents, and the $K$-module of morphisms $\operatorname{Hom}_{A}\left(e_{i}, e_{j}\right)$ from $e_{i}$ to $e_{j}$ is equal $e_{j} A e_{i}$. Then $A^{\text {op }}$ is the opposite $K$-category to $A$, and so $\operatorname{Hom}_{A^{\text {op }}}\left(e_{i}, e_{j}\right)=e_{i} A e_{j}$ for all $1 \leq i, j \leq s$.

Now let $I$ be an ideal of $A, B=A / I$, and $e=e_{1}+\ldots+e_{t}$, for some $t \leq s$, a residual identity of $B$. Assume that $I$ satisfies the conditions (1) and (2), introduced in Section 1, and the following condition:

(3) The canonical algebra homomorphism $\varrho: e A e \rightarrow e A e / e I e$ splits.

We claim that there is a retraction $\iota$ of $\varrho$ (an algebra homomorphism $\iota: e A e / e I e \rightarrow$ $e A e$ with $\rho \iota=1$ on $e A e / e I e)$ such that $\iota\left(e_{i}\right)=e_{i}$ for any $1 \leq i \leq t$. Indeed, let $\iota_{0}: e A e / e I e \rightarrow e A e$ be a retraction of $\varrho$. Then $e_{i}+e I e=\iota_{0}\left(e_{i}\right)$ for any $1 \leq i \leq t$. Since $\left\{\iota_{0}\left(e_{i}\right) \mid 1 \leq i \leq t\right\}$ is a complete set of primitive orthogonal idempotents of $e A e$ and $(e I e)^{2}=0$, by the imposed condition (1), we deduce from the Krull-Schmidt theorem that there is an invertible element $u$ of $e A e$ such that $e_{i}=u^{-1} \iota_{0}\left(e_{i}\right) u$ for all $1 \leq i \leq t$. Put $\iota\left(e_{i} b e_{j}\right)=u^{-1} \iota_{0}\left(e_{i} b e_{j}\right) u$ for all $1 \leq i, j \leq t$ and $b \in e A e / e I e$. Then $\iota: e A e / e I e \rightarrow e A e$ is an algebra homomorphism such that $\varrho \iota=1$ on $e A e / e I e$ and $\iota\left(e_{i}\right)=e_{i}$ for any $1 \leq i \leq t$. Hence, under the above assumptions, we may assume that $\iota$ is a retraction of $\varrho$ such that $\iota\left(e_{i} \bar{a} e_{j}\right)=e_{i} \iota(\bar{a}) e_{j}$ for all $1 \leq i, j \leq t$ and $\bar{a}=a+e I e \in e A e / e I e$. Further, by the canonical algebra homomorphisms $A \rightarrow B \rightarrow e A e / e I e$, both $B$ and $e A e / e I e$ can be considered as $A$-algebras. Hence $B$ and $e A e / e I e$ are isomorphic $A$-algebras, and from now on we shall identify them. Moreover, we shall identify $B$ with $\iota(B)$.

Consider now the repetitive algebra

$$
\widehat{B}=\left[\begin{array}{cccccc}
\ddots & \ddots & & & & \\
& B_{m-1} & Q_{m-1} & & & \\
& & B_{m} & Q_{m} & & \\
& & & B_{m+1} & Q_{m+1} & \\
& & & & \ddots & \ddots
\end{array}\right]
$$

of $B$ with $B_{m}=B$ and $Q_{m}=D(B)$ for all $m \in \mathbb{Z}$. We denote by $\left\{e_{m, i} \mid 1 \leq i \leq\right.$ $t, m \in \mathbb{Z}\}$ the canonical set of primitive orthogonal idempotents of $\widehat{B}$ induced by $\left\{e_{i} \mid 1 \leq i \leq t\right\}$. Hence, for $1 \leq i \leq t$ and $m \in \mathbb{Z}$, we have

$$
e_{m, i} \widehat{B}=e_{i} B \oplus e_{i} D(B) \quad \text { and } \quad e_{m, i} \widehat{B} e_{m+1, j}=e_{i} D(B) e_{j}
$$


Applying the Nakayama automorphism $\nu$ of $A$ to the idempotents $e_{i}, 1 \leq i \leq t$, we may get an idempotent $\nu\left(e_{i}\right)=e_{\nu(i)}$, with $\nu(i)>t$, equivalently $e_{\nu(i)} \in I$, and hence $e_{\nu(i)}$ is a zero element of $B$. Therefore, we introduce the convention that $e_{n, i} \widehat{B}=0=\widehat{B} e_{n, i}$ for $i>t$ and $n \in \mathbb{Z}$.

We may clearly consider $\widehat{B}$ as a locally bounded $K$-category whose set of objects is equal $\left\{e_{m, i} \mid i \leq t, m \in \mathbb{Z}\right\}$ and $\operatorname{Hom}_{\widehat{B}}\left(e_{n, i}, e_{m, j}\right)=e_{m, j} \widehat{B} e_{n, i}$ for all $1 \leq i, j \leq t$, $m, n \in \mathbb{Z}$. The canonical shifting automorphism $\nu_{\widehat{B}}: \widehat{B} \rightarrow \widehat{B}$ with $\nu_{\widehat{B}}\left(e_{m, i}\right)=$ $e_{m+1, i}$ for all $m \in \mathbb{Z}, 1 \leq i \leq t$, is called the Nakayama automorphism of $\widehat{B}$. An automorphism $\varphi$ of $\widetilde{B}$ is said to be positive (respectively, strictly positive) if for each object $e_{m, i}$ of $\widehat{B}$, there are an integer $p \geq m$ (respectively, $p>m$ ) and $1 \leq j \leq t$ such that $\varphi\left(e_{m, i}\right)=e_{p, j}$. Hence, a positive automorphism of $\widehat{B}$ is a shift of $\widehat{B}$ in the same direction as $\nu_{\widehat{B}}$.

The main purpose of this section is to prove the following theorem and some its consequences.

Theorem 3.1. Let $A$ be a basic and connected selfinjective artin algebra, $I$ an ideal of $A, B=A / I$, and e a residual identity of $B$. Assume that the following conditions are satisfied:

(1) $\widetilde{e}(I e I) \widetilde{e}=0$.

(2) $\nu^{-}(e) I e$ is an injective cogenerator in $\bmod e A e / e I$ e and $e I \nu(e)$ is an injective cogenerator in $\bmod (e A e / e I e)^{\mathrm{op}}$.

(3) The canonical algebra epimorphism $\varrho: e A e \rightarrow e A e / e I e$ splits.

Then there is a Galois covering $F: \widehat{B} \rightarrow \widetilde{e} A \widetilde{e}$ with the Galois group $G$ infinite cyclic generated by an automorphism $\varphi \nu_{\widehat{B}}$, where $\varphi$ is a positive automorphism of $\widehat{B}$.

We divide the proof of the above theorem into several steps. First we define a functor $F: \widehat{B} \rightarrow \widetilde{e} A \widetilde{e}$ as follows:

For each object $e_{m, i}$ of $\widehat{B}$ we put $F\left(e_{m, i}\right)=e_{\nu^{m}(i)}$. Then:

$$
F: \operatorname{Hom}_{\widehat{B}}\left(e_{m, i}, e_{n, j}\right) \rightarrow \operatorname{Hom}_{\tilde{e} A \tilde{e}}\left(F\left(e_{m, i}\right), F\left(e_{n, j}\right)\right)=\operatorname{Hom}_{\tilde{e} A \widetilde{e}}\left(e_{\nu^{m}}(i), e_{\nu^{n}(j)}\right)
$$

is defined as follows:

(a) If $m=n$, then $F$ is the composition

$$
e_{m, j} \widehat{B} e_{m, i}=e_{j} B e_{i} \stackrel{\iota}{\longrightarrow} e_{j} A e_{i} \stackrel{\nu^{m}}{\longrightarrow} e_{\nu^{m}(j)} A e_{\nu^{m}(i)} .
$$

(b) If $n=m-1$, then $F$ is the composition

$e_{m-1, j} \widehat{B} e_{m, i}=e_{j} D(B) e_{i} \stackrel{\Theta_{r}^{-1}}{\sim} e_{\nu^{-}(j)} I e_{i} \longrightarrow e_{\nu^{-}(j)} A e_{i} \stackrel{\nu^{m}}{\longrightarrow} e_{\nu^{m-1}(j)} A e_{\nu^{m}(i)}$.

(c) $F$ is zero for $n \neq m$ and $n \neq m-1$.

In the case when $n=m-1$, we have used the isomorphism $\Theta_{r}: A \rightarrow{ }_{\nu} D(A)$. But, by Lemma 2.9 , the composition in (b) equals the composition

$$
e_{m-1, j} \widehat{B} e_{m, i}=e_{j} D(B) e_{i} \stackrel{\Theta_{l}^{-1}}{\sim} e_{j} I e_{\nu(i)} \subset e_{j} A e_{\nu(i)} \stackrel{\nu^{m-1}}{\longrightarrow} e_{\nu^{m-1}(j)} A e_{\nu^{m}(i)} .
$$

Using the fact that $\iota$ and $\nu$ are algebra homomorphisms and $\Theta_{r}$ defines a right $e A e$ homomorphism from $D(B) e$ to $\nu^{-}(e) I e$, it is easy to check that $F$ defined above is a functor from $\widehat{B}$ to $\widetilde{e} A \widetilde{e}$.

Lemma 3.2. $F: \widehat{B} \rightarrow \widetilde{e} A \widetilde{e}$ is a covering functor. 
Proof. We have to show that for any $1 \leq i, j \leq t$ and $m, n \in \mathbb{Z}$, the maps

$$
\bigoplus_{k \in \mathbb{Z}} \operatorname{Hom}_{\widehat{B}}\left(e_{m, i}, e_{k, \nu^{n-k}(j)}\right) \rightarrow \operatorname{Hom}_{\tilde{e} A \tilde{e}}\left(e_{\nu^{m}(i)}, e_{\nu^{n}(j)}\right)
$$

and

$$
\bigoplus_{k \in \mathbb{Z}} \operatorname{Hom}_{\widehat{B}}\left(e_{k, \nu^{m-k}(i)}, e_{n, j}\right) \rightarrow \operatorname{Hom}_{\tilde{e} A \tilde{e}}\left(e_{\nu^{m}(i)}, e_{\nu^{n}(j)}\right),
$$

induced by $F$, are isomorphisms. By definition of $\widehat{B}$ we get

$$
\begin{aligned}
\bigoplus_{k \in \mathbb{Z}} \operatorname{Hom}_{\widehat{B}}\left(e_{m, i}, e_{k, \nu^{n-k}(j)}\right) & \\
=\operatorname{Hom}_{\widehat{B}}\left(e_{m, i}, e_{m, \nu^{n-m}}(j)\right. & \oplus \operatorname{Hom}_{\widehat{B}}\left(e_{m, i}, e_{m-1, \nu^{n-m+1}(j)}\right) .
\end{aligned}
$$

Moreover, we have the isomorphisms

$$
\begin{aligned}
& e_{\nu^{n-m}(j)} B e_{i} \oplus e_{\nu^{n-m+1}(j)} D(B) e_{i} \\
& 1 \oplus \Theta_{r}^{-1} \downarrow \sim \sim \\
& e_{\nu^{n-m}(j)} B e_{i} \oplus e_{\nu^{n-m}(j)} I e_{i}= e_{\nu^{n-m}(j)} A e_{i} \\
& \nu^{m} \stackrel{\sim}{\sim} \\
& e_{\nu^{n}(j)} A e_{\nu^{m}(j)}
\end{aligned}
$$

where, in the case when $\nu^{n-m}(j)>t, e_{\nu^{n-m}(j)} B e_{i}=0$, by our convention, and $e_{\nu^{n-m}(j)} I e_{i}=e_{\nu^{n-m}(j)} A e_{i}$ by the fact that $1-e \in I$. This proves that the first map is an isomorphism. By making use of $\Theta_{l}$ instead of $\Theta_{r}$ in the considerations above, we prove similarly that the second map is also an isomorphism.

Our next aim is to define an infinite cyclic group $G$ of automorphisms of $\widehat{B}$ acting freely on the objects of $\widehat{B}$, and then to show that $F: \widehat{B} \rightarrow \widetilde{e} A \widetilde{e}$ is a Galois covering with the Galois group $G$.

For each $1 \leq i \leq t$, we put

$$
\eta(i)=\min \left\{k>0 \mid \nu^{k}(i) \leq t\right\}
$$

and define a map $g: \mathbb{Z} \times\{1, \ldots, t\} \rightarrow \mathbb{Z} \times\{1, \ldots, t\}$ by

$$
g(m, i)=\left(m-\eta(i), \nu^{\eta(i)}(i)\right)
$$

for all $(m, i) \in \mathbb{Z} \times\{1, \ldots, t\}$. Observe that $g$ is a bijection, because the action of $\nu$ on the set $\{1, \ldots, s\}$ has finite order, and, for $1 \leq i, j \leq t, \nu^{\eta(i)}(i)=\nu^{\eta(j)}(j)$ implies $i=j$. This allows to define a bijection on the objects of $\widehat{B}$, denoted again by $g$, by

$$
g\left(e_{m, i}\right)=e_{g(m, i)}
$$

for all $(m, i) \in \mathbb{Z} \times\{1, \ldots, t\}$.

Lemma 3.3. For each pair $(m, i) \in \mathbb{Z} \times\{1, \ldots, t\}$, the cyclic group generated by $g$ acts transitively on the fiber $F^{-1}\left(e_{\nu^{m}}(i)\right)$.

Proof. Fix $(m, i) \in \mathbb{Z} \times\{1, \ldots, t\}$. Then

$$
F^{-1}\left(e_{\nu^{m}(i)}\right)=\left\{e_{m-k, \nu^{k}(i)} \mid k \in \mathbb{Z}, 1 \leq \nu^{k}(i) \leq t\right\} .
$$


It suffices to show that for any $k, n \in \mathbb{Z}$ with $k<n$ and $1 \leq \nu^{n}(i) \leq t$, there exists $l \in \mathbb{Z}$ such that $g^{l}\left(e_{m-k, \nu^{k}(i)}\right)=e_{m-n, \nu^{n}(i)}$. We define a sequence of integers

$$
k=s_{0}<s_{1}<s_{2}<\ldots
$$

inductively by $s_{p+1}=s_{p}+\eta\left(\nu^{s_{p}}(i)\right)$. Observe that

$$
g\left(e_{m-s_{p}, \nu^{s_{p}}(i)}\right)=e_{m-s_{p+1}, \nu^{s_{p+1}}(i)}
$$

for all $p \geq 0$. Further, $\nu^{q}(i)>t$ for any integer $q$ with $s_{p}<q<s_{p+1}$. Moreover, by our assumptions, we have $k<n$ and $\nu^{n}(i) \leq t$. Hence, $n=s_{l}$ for some $l>0$, and consequently we obtain $g^{l}\left(e_{m-k, \nu^{k}(i)}\right)=e_{m-n, \nu^{n}(i)}$.

We shall now extend the bijection $g$ on the objects of $\widehat{B}$ to an automorphism of $\widehat{B}$ such that $F g=F$. For this purpose, we prove several technical lemmas. Observe first that for $(m, i),(n, j) \in \mathbb{Z} \times\{1, \ldots, t\}$ we have by Lemma 3.2 the following isomorphisms:

$$
\begin{aligned}
e_{\nu^{n}(j)} A_{\nu^{m}(i)} & \simeq \bigoplus_{k} \operatorname{Hom}_{\widehat{B}}\left(e_{0, \nu^{m}(i)}, e_{n-k, \nu^{k}(i)}\right) \\
& \simeq \operatorname{Hom}_{\widehat{B}}\left(e_{0, \nu^{m}(i)}, e_{0, \nu^{m}(j)}\right) \oplus \operatorname{Hom}_{\widehat{B}}\left(e_{0, \nu^{m}(i)}, e_{-1, \nu^{m+1}(j)}\right) \\
& \simeq e_{\nu^{m}(j)} B e_{\nu^{m}(i)} \oplus D\left(e_{\nu^{m}(i)} B e_{\nu^{m+1}(j)}\right)
\end{aligned}
$$

and

$$
\begin{aligned}
e_{\nu^{n}(j)} A e_{\nu^{m}(i)} & \simeq \bigoplus_{k} \operatorname{Hom}_{\widehat{B}}\left(e_{m-k, \nu^{k}(i)}, e_{0, \nu^{n}(j)}\right) \\
& \simeq \operatorname{Hom}_{\widehat{B}}\left(e_{0, \nu^{n}(i)}, e_{0, \nu^{n}(j)}\right) \oplus \operatorname{Hom}_{\widehat{B}}\left(e_{1, \nu^{n-1}(i)}, e_{0, \nu^{n}(j)}\right) \\
& \simeq e_{\nu^{n}(j)} B e_{\nu^{n}(i)} \oplus D\left(e_{\nu^{n-1}(i)} B e_{\nu^{n}(j)}\right) .
\end{aligned}
$$

Lemma 3.4. Assume $1 \leq i, j \leq t$ and $e_{j} B e_{i}$ is nonzero. Then $\eta(j)=\eta(i)$ or $\eta(j)=\eta(i)+1$.

Proof. Note that for any $k \in \mathbb{Z}$ we have the composed epimorphism

$$
e_{\nu^{k}(j)} A e_{\nu^{k}(i)} \stackrel{\nu^{-k}}{\longrightarrow} e_{j} A e_{i} \longrightarrow e_{j} B e_{i},
$$

and hence $e_{\nu^{k}(j)} A e_{\nu^{k}(i)} \neq 0$, because $e_{j} B e_{i} \neq 0$ by our assumption.

Consider first the case $\eta(j)=1$. Clearly, $e_{j} B e_{i} \neq 0$ implies that $e_{j} I e_{i}$ is a proper subset of $e_{j} A e_{i}$. Further, by Lemma 2.8, we have $e_{\nu(j)} D(B) e_{i} \simeq e_{j} I e_{i}$. Applying now $(* *)$ for $n=1$ we conclude that $e_{\nu(j)} B e_{\nu(i)} \neq 0$, and so $\eta(i)=1$.

Assume now that $\eta(j)>1$. Let $m=\eta(i)$ and $n=\eta(j)$. Since $e_{\nu^{n}(j)} A e_{\nu^{n}(i)} \neq 0$, applying $(* *)$, we get $e_{\nu^{n}(j)} B e_{\nu^{n}(i)} \neq 0$ or $e_{\nu^{n-1}(i)} B e_{\nu^{n}(j)} \neq 0$. Hence $\eta(i) \leq \eta(j)$. Moreover, since $e_{\nu^{m}(j)} A e_{\nu^{m}(i)} \neq 0$, applying $(*)$, we deduce that $e_{\nu^{m}(j)} B e_{\nu^{m}(i)} \neq 0$ or $e_{\nu^{m}(i)} B e_{\nu^{m+1}(j)} \neq 0$, and so $\eta(j) \leq \eta(i)+1$. Therefore, we get $\eta(i) \leq \eta(j) \leq$ $\eta(i)+1$, which proves our claim.

Lemma 3.5. Assume $1 \leq i, j \leq t$ and $\eta(i)=\eta(j)=n$. Then

$$
e_{\nu^{n}(j)} \nu^{n}(B) e_{\nu^{n}(i)}=e_{\nu^{n}(j)} B e_{\nu^{n}(i)}
$$

and

$$
e_{\nu^{n-1}(i)} \nu^{n}(I) e_{\nu^{n}(j)}=e_{\nu^{n-1}(i)} I e_{\nu^{n}(j)} .
$$


Proof. First we show the second equality. It follows from Lemma 2.9 that $e_{i} \nu(I) e_{\nu(j)}$ $=e_{i} I e_{\nu(j)}$ which is the second equality for $n=1$. Suppose now $n>1$. Then $\nu^{r}(j)>$ $t$ for $1 \leq r \leq n-1$, by definition of $\eta(j)$. We then get $e_{i} A e_{\nu(j)}=e_{i} I e_{\nu(j)}$. Further, by Lemma 2.9, we have $e_{\nu^{n-1}(i)} \nu^{n-1}(I) e_{\nu^{n}(j)}=e_{\nu^{n-1}(i)} \nu^{n}(I) e_{\nu^{n}(j)}$. Hence, $e_{\nu^{n-1}(i)} \nu^{n}(I) e_{\nu^{n}(j)}=e_{\nu^{n-1}(i)} A e_{\nu^{n}(j)}$. On the other hand, $\nu^{n-1}(i)>t$, because $n=$ $\eta(i)$, and so $e_{\nu^{n-1}(i)} I e_{\nu^{n}(j)}=e_{\nu^{n-1}(i)} A e_{\nu^{n}(j)}$. Consequently, $e_{\nu^{n-1}(i)} \nu^{n}(I) e_{\nu^{n}(j)}$ $=e_{\nu^{n-1}(i)} I e_{\nu^{n}(j)}$. Now, applying $\nu^{-n}$ to the proved equality, we have $e_{\nu^{-}(i)} I e_{j}=$ $e_{\nu^{-}(i)} \nu^{-n}(I) e_{j}$. From Lemma 2.7 we have $D\left(e_{j} B e_{i}\right)=D\left(e_{j} \nu^{-n}(B) e_{i}\right)$, and so applying $D\left(\nu^{n}\right)$ we get $D\left(\nu^{n}\left(e_{j} B e_{i}\right)\right)=D\left(e_{\nu^{n}(j)} B e_{\nu^{n}(i)}\right)$. Hence we obtain the first required equality.

Lemma 3.6. Assume $1 \leq i, j \leq t, \eta(j)=\eta(i)+1, \eta(i)=n$. Then

$$
e_{\nu^{n}(j)} \nu^{n}(B) e_{\nu^{n}(i)}=e_{\nu^{n}(j)} I e_{\nu^{n}(i)}
$$

and

$$
e_{\nu^{n}(i)} \nu^{n}(I) e_{\nu^{n+1}(j)}=e_{\nu^{n}(i)} B e_{\nu^{n+1}(j)} .
$$

Proof. Applying the isomorphisms from Lemma 2.7 as in the proof of Lemma 3.5, we deduce that the second required equality follows from the first one. Hence we have to prove only the first equality.

Since $\eta(j)>n$, we have $e_{\nu^{n}(j)} \in I$, and so $e_{\nu^{n}(j)} I e_{\nu^{n}(i)}=e_{\nu^{n}(j)} A e_{\nu^{n}(i)}$. Moreover, $e_{\nu(j)} \in I$ because $\eta(j)>1$. We claim that $e_{j} I e_{i}=0$. Indeed, suppose that $e_{j} I e_{i} \neq 0$. Then $\operatorname{soc}\left(e_{j} I e_{i} A\right) \simeq \operatorname{top}\left(e_{\nu(j)} A\right)$ as right $A$-modules, and hence $e_{j} I e_{i} A e_{\nu(j)} \neq 0$. But this implies $\widetilde{e}(I e I) \widetilde{e} \neq 0$ because $e_{\nu(j)} \in I$, contradicting the assumption (1). Therefore, $e_{j} I e_{i}=0$ and $e_{j} A e_{i}=e_{j} B e_{i}$. Applying $\nu^{n}$ we get $e_{\nu^{n}(j)} A e_{\nu^{n}(i)}=e_{\nu^{n}(j)} \nu^{n}(B) e_{\nu^{n}(i)}$. Since $e_{\nu^{n}(j)} \in I$, this gives the required equality $e_{\nu^{n}(j)} \nu^{n}(B) e_{\nu^{n}(i)}=e_{\nu^{n}(j)} I e_{\nu^{n}(i)}$.

Fix now two objects $e_{m, i}$ and $e_{n, j}$ of $\widehat{B}$ and consider the $K$-linear homomorphisms

$$
\begin{aligned}
F_{1}: \operatorname{Hom}_{\widehat{B}}\left(e_{m, i}, e_{n, j}\right) & \rightarrow F\left(\operatorname{Hom}_{\widehat{B}}\left(e_{m, i}, e_{n, j}\right)\right), \\
F_{2}: \operatorname{Hom}_{\widehat{B}}\left(g\left(e_{m, i}\right), g\left(e_{n, j}\right)\right) & \rightarrow F\left(\operatorname{Hom}_{\widehat{B}}\left(g\left(e_{m, i}\right), g\left(e_{n, j}\right)\right)\right)
\end{aligned}
$$

induced by the functor $F: \widehat{B} \rightarrow \widetilde{e} A \widetilde{e}$. It follows from definition of $F$ that both $F_{1}$ and $F_{2}$ are monomorphisms.

We shall prove that the images of $F_{1}$ and $F_{2}$ are equal, and then

$$
F_{2}^{-1} F_{1}: \operatorname{Hom}_{\widehat{B}}\left(e_{m, i}, e_{n, j}\right) \rightarrow \operatorname{Hom}_{\widehat{B}}\left(g\left(e_{m, i}, e_{n, j}\right)\right)
$$

will define the required action of $g$ on $\operatorname{Hom}_{\widehat{B}}\left(e_{m, i}, e_{n, j}\right)$. We have several cases to consider.

Assume first that $\operatorname{Hom}_{\widehat{B}}\left(e_{m, i}, e_{n, j}\right) \neq 0$. Then $n=m$ or $n=m-1$.

(i) Suppose $n=m$. Then $e_{j} B e_{i}=\operatorname{Hom}_{\widehat{B}}\left(e_{m, i}, e_{m, j}\right) \neq 0$ and hence $\eta(j)=\eta(i)$ or $\eta(j)=\eta(i)+1$, by Lemma 3.4. In the case when $\eta(j)=\eta(i)$, by Lemma 3.5, we have $e_{\nu^{k}(j)} \nu^{k}(B) e_{\nu^{k}(i)}=e_{\nu^{k}(j)} B e_{\nu^{k}(i)}$, where $k=\eta(i)$. Then, applying $\nu^{m-k}$, we obtain $e_{\nu^{m}(j)} \nu^{m}(B) e_{\nu^{m}(i)}=e_{\nu^{m}(j)} \nu^{m-k}(B) e_{\nu^{m}(i)}$. This shows that $\operatorname{Im} F_{1}=\operatorname{Im} F_{2}$ in this case. In the case when $\eta(j)=\eta(i)+1$, applying Lemma 3.6, we have $e_{\nu^{k}(j)} \nu^{k}(B) e_{\nu^{k}(i)}=e_{\nu^{k}(j)} I e_{\nu^{k}(i)}$, where again $k=\eta(i)$. Hence $e_{\nu^{m}(j)} \nu^{m}(B) e_{\nu^{m}(i)}=$ $e_{\nu^{m}(j)} \nu^{m-k}(I) e_{\nu^{m}(i)}$, and consequently $\operatorname{Im} F_{1}=\operatorname{Im} F_{2}$.

(ii) Suppose $n=m-1$. Then $e_{j} D(B) e_{i}=\operatorname{Hom}_{\widehat{B}}\left(e_{m, i}, e_{m-1, j}\right) \neq 0$, and hence, by Lemma 3.4, $\eta(i)=\eta(j)$ or $\eta(i)=\eta(j)+1$. For $\eta(i)=\eta(j)$, it follows from 
Lemma 3.5 that $e_{\nu^{k-1}(i)} \nu^{k}(I) e_{\nu^{k}(j)}=e_{\nu^{k-1}(i)} I e_{\nu^{k}(i)}$, and so $e_{\nu^{m-1}(i)} \nu^{m}(I) e_{\nu^{m}(j)}$ $=e_{\nu^{m-1}(i)} \nu^{m-k}(I) e_{\nu^{m}(i)}$, where $k=\eta(j)$. For $\eta(i)=\eta(j)+1$, it follows from Lemma 3.6 that $e_{\nu^{k}(j)} \nu^{k}(I) e_{\nu^{k+1}(i)}=e_{\nu^{k}(j)} B e_{\nu^{k+1}(j)}$, and so $e_{\nu^{m-1}(j)} \nu^{m-1}(I) e_{\nu^{m}(i)}$ $=e_{\nu^{m-1}(j)} \nu^{m-(k+1)}(B) e_{\nu^{m}(j)}$, where again $k=\eta(j)$. Hence, in both cases we have $\operatorname{Im} F_{1}=\operatorname{Im} F_{2}$. We have also proved that $\operatorname{Hom}_{\widehat{B}}\left(e_{m, i}, e_{n, j}\right) \neq 0$ implies $\operatorname{Hom}_{\widehat{B}}\left(g\left(e_{m, i}\right), g\left(e_{n, j}\right)\right) \neq 0$. Assume next that $\operatorname{Hom}_{\widehat{B}}\left(e_{m, i}, e_{n, j}\right)=0$. We have to show that also

$$
\operatorname{Hom}_{\widehat{B}}\left(e_{g(m, i)}, e_{g(n, j)}\right)=\operatorname{Hom}_{\widehat{B}}\left(g\left(e_{m, i}\right), g\left(e_{n, j}\right)\right)=0 .
$$

Suppose $\left.\operatorname{Hom}_{\widehat{B}}\left(e_{g(m, i)}, e_{g(n, j)}\right)\right) \neq 0$. Then $\operatorname{Hom}_{\widehat{B} \text { op }}\left(e_{g(n, j)}, e_{g(m, i)}\right) \neq 0$. Observe now that the conditions (1), (2) and (3) imposed on $A$ are left-right symmetric. For each $1 \leq i \leq t$, put

$$
\xi(i)=\min \left\{k>0 \mid \nu^{-k}(i) \leq t\right\}
$$

and define $h: \mathbb{Z} \times\{1, \ldots, t\} \rightarrow \mathbb{Z} \times\{1, \ldots, t\}$ by

$$
h(m, i)=\left(m+\xi(i), \nu^{-\xi(i)}(i)\right)
$$

for all $(m, i) \in \mathbb{Z} \times\{1, \ldots, t\}$. By the above remark, applied now to left $\widehat{B}$-modules (right $\widehat{B}^{\mathrm{op}}$-modules) we deduce that $\operatorname{Hom}_{\widehat{B}^{\text {op }}}\left(e_{g(n, j)}, e_{g(m, i)}\right) \neq 0$ implies

$$
\operatorname{Hom}_{\widehat{B}_{\mathrm{op}}}\left(e_{h g(n, j)}, e_{h g(m, i)}\right)=\operatorname{Hom}_{\widehat{B}_{\mathrm{op}}}\left(h\left(e_{g(n, j)}\right), h\left(e_{g(m, i)}\right)\right) \neq 0 .
$$

Hence we have $\operatorname{Hom}_{\widehat{B}}\left(e_{h g(m, i)}, e_{h g(n, j)}\right) \neq 0$. But $\xi\left(\nu^{\eta(i)}(i)\right)=\eta(i)$ for any $1 \leq i \leq$ $t$, and so $h g(m, i)=(m, i)$ and $h g(n, j)=(n, j)$. Therefore, we get $\operatorname{Hom}_{\widehat{B}}\left(e_{m, i}, e_{n, j}\right)$ $\neq 0$ which contradicts our assumption. This shows that indeed $\operatorname{Hom}\left(e_{m, i}, e_{n, j}\right)=0$ implies $\operatorname{Hom}_{\widehat{B}}\left(g\left(e_{m, i}\right), g\left(e_{n, j}\right)\right)=0$, and we are done.

Denote now by $G$ the group of $K$-linear automorphisms of $\widehat{B}$ generated by $g$. Clearly, $G$ is infinite cyclic, $F g^{\prime}=F$ for any element $g^{\prime}$ of $G$, and, by Lemma 3.3, $G$ acts transitively on the fibres $F^{-1}\left(e_{\nu^{m}(i)}\right)$ of the covering $F: \widehat{B} \rightarrow \widetilde{e} A \widetilde{e}$. Therefore, $F$ is a Galois covering with the Galois group $G$ and $\widetilde{e} A \widetilde{e} \simeq \widehat{B} / G$. Finally, observe that $g^{-1}$ is defined on the objects of $\widehat{B}$ by the formula

$$
g^{-1}\left(e_{m, i}\right)=e_{m+\eta(i), \nu^{-\eta(i)}(i)}
$$

for all $(m, i) \in \mathbb{Z} \times\{1, \ldots, t\}$. Since $\nu(i) \geq 1$ for any $1 \leq i \leq t$, we infer that $g^{-1}=$ $\varphi \nu_{\widehat{B}}$ for some positive automorphism $\varphi$ of $\widehat{B}$ with $\varphi\left(e_{m, i}\right)=e_{m+\eta(i)-1, \nu^{-\eta(i)}(i)}$, and obviously $G$ is generated by $\varphi \nu_{\widehat{B}}$. This finishes the proof of Theorem 3.1.

We are now able to prove a criterion for a selfinjective artin algebra $A$ to be of the form $\widehat{B} / G$. Before, we recall the following proposition proved in [23, Proposition 2.3].

Proposition 3.7. Let $A$ be a selfinjective artin algebra, $I$ an ideal of $A, B=A / I$, $e$ a residual identity of $B$, and assume that $I e I=0$. Then the following conditions are equivalent:

(i) Ie is an injective cogenerator in $\bmod B$.

(ii) $e I$ is an injective cogenerator in $\bmod B^{\mathrm{op}}$.

(iii) $e I=r_{A}(I)$.

(iv) $I e=l_{A}(I)$.

Moreover, under these conditions, $\operatorname{soc} A \subseteq I$ and $e I e=l_{e A e}(I)=r_{e A e}(I)$. 
Theorem 3.8. Let $A$ be a basic and connected selfinjective artin algebra, $I$ an ideal of $A, B=A / I$, and $e$ a residual identity of $B$. Assume $I e I=0, I e=l_{A}(I)$ and the canonical algebra epimorphism eAe $\rightarrow$ eAe/eIe splits. Then there is a Galois covering $F: \widehat{B} \rightarrow A$ with the Galois group $G$ infinite cyclic generated by an automorphism $\varphi \nu_{\widehat{B}}$ of $\widehat{B}$, where $\varphi$ is a positive automorphism of $\widehat{B}$.

Proof. We identify again $B$ with $e A e / e I e$. Since $I e I=0$, it follows from Corollary 2.4 that $\widetilde{e}=1$. Moreover, by the above proposition $I e$ is an injective cogenerator in $\bmod B$ and $e I$ is an injective cogenerator in $\bmod B^{\mathrm{op}}$. Clearly, then $I e=\nu^{-}(e) I e$ and $e I=e I \nu(e)$ (see also Lemma 2.6). Therefore, $A$ satisfies the conditions (1), (2) and (3) of Theorem 3.1, and this finishes the proof.

We end this section with the following consequence of the above results.

Corollary 3.9. Let $A$ be a basic and connected selfinjective artin algebra, $I$ an ideal of $A$, and $B=A / I$. Assume $I^{2}=0, I$ is an injective cogenerator as left and right $B$-module, and the canonical algebra epimorphism $A \rightarrow A / I$ splits. Then there is a Galois covering $F: \widehat{B} \rightarrow A$ with the Galois group generated by $\varphi \nu_{\widehat{B}}$, where (in our notations) $\varphi$ is an automorphism of $\widehat{B}$ such that $\varphi\left(e_{m, i}\right)=e_{m, \nu^{-}}(i)$ for any integer $m$ and $1 \leq i \leq s$.

Proof. Since $I$ is contained in the radical of $A, 1=\sum_{i=1}^{s} e_{i}$ is a residual identity of $B=A / I$. Hence, $e_{\nu(i)} \in B$ for any $1 \leq i \leq s$. This implies that $\eta(i)=1$ for any $1 \leq i \leq s$. Hence, by definition of $g$, we have $g(m, i)=(m-1, \nu(i))$ and $\varphi\left(e_{m, i}\right)=e_{m, \nu^{-}(i)}$.

Remark 3.10. Let $B$ be any basic and connected artin algebra and $A=B \ltimes D(B)$ the trivial extension of $B$ by $D(B)$. Then the Nakayama automorphism $\nu$ of $A$ is the identity and $I=D(B)$ satisfies the conditions of the above corollary. Hence Corollary 3.9 generalizes the well-known fact that the natural functor $\widehat{B} \rightarrow B \ltimes$ $D(B)=A$ is a Galois covering with the Galois group generated by $\nu_{\widehat{B}}$.

\section{SelfinjeCtive Algebras With Deforming ideALS}

In our paper [26] we investigated ring-theoretic properties of certain ideals of selfinjective artin algebras, called deforming ideals. Recall from [[26], (2.1)] that, if $I$ is an ideal of a selfinjective artin algebra and $e$ a residual identity of $B=A / I$, then $I$ is called deforming if the ordinary quiver $Q_{B}$ of $B$ has no oriented cycles and $l_{e A e}(I)=e I e=r_{e A e}(I)$. An important class of deforming ideals is formed by the ideals $I$ satisfying the conditions: $Q_{B}$ has no oriented cycles, $I e I=0$ and $I e$ is an injective cogenerator in $\bmod B$ (see Proposition 3.7). In this section, applying the main results of Section 3 and [26], we determine the structure of some classes of selfinjective artin algebras having deforming ideals.

Theorem 4.1. Let $A$ be a basic and connected selfinjective artin algebra over a commutative artin ring $K$. Let $I$ be an ideal of $A, B=A / I$, and $e$ a residual identity of $B$. Assume that the ordinary quiver $Q_{B}$ of $B$ has no oriented cycles, $I e I=0$ and $I e$ is an injective cogenerator in $\bmod B$. Then $A$ is socle equivalent to an algebra $\widehat{B} / G$ where $G$ is an infinite cyclic group of automorphisms of $\widehat{B}$ generated by $\varphi \nu_{\widehat{B}}$, for some positive automorphism $\varphi$ of $\widehat{B}$. Moreover, if $K$ is an algebraically closed field, then $A$ is isomorphic to $\widehat{B} / G$. 
Proof. Assume first that $K$ is an algebraically closed field. Then it follows from [26, Theorem 3.2] that the Hochschild cohomology $H^{2}(e A e / e I e, e I e)$ vanishes, and hence the canonical algebra epimorphism $e A e \rightarrow e A e / e I e$ splits. Therefore, applying Theorem 3.8, we deduce that $A=\widehat{B} / G$, where $G$ is an infinite cyclic group generated by $\varphi \nu_{\widehat{B}}$, for some positive automorphism $\varphi$ of $\widehat{B}$.

If $K$ is an arbitrary commutative artin ring (even a field), then the canonical algebra epimorphism $e A e \rightarrow e A e / e I e$ does not necessarily split (see example below). But we may replace $A$ by a socle equivalent selfinjective algebra $A[I]$ satisfying the conditions of Theorem 3.8. Indeed, following [26, Section 4], consider the algebra $A[I]$ whose $K$-linear structure is that of $B \oplus I$ and the multiplication is given by

$$
(b, x)\left(b^{\prime}, x\right)=\left(b b^{\prime}, b x^{\prime}+x b^{\prime}+x x^{\prime}\right)
$$

for all $b, b^{\prime} \in B$ and $x, x^{\prime} \in I$. Then $A[I]$ is a basic and connected selfinjective artin $K$-algebra, $I=\{(0, x) \mid x \in I\}$ is an ideal of $A[I]$, $e$ a residual identity of $B=A[I] / I, I e I=0, I e=l_{A[I]}(I)$, and clearly the canonical algebra epimorphism $e A[I] e \rightarrow e A[I] e / e I e$ splits. Hence, by Theorem 3.8, $A[I]$ is isomorphic to an algebra $\widehat{B} / G$, where $G$ is an infinite cyclic group generated by $\varphi \nu_{\widehat{B}}$, for some positive automorphism $\varphi$ of $\widehat{B}$. Moreover, it is shown in [26, Theorem 4.1] that $A$ and $A[I]$ are socle equivalent. This finishes the proof.

Example 4.2. Let $K \subseteq L$ be a finite field extension of a field $K$ of characteristic 2 such that $H^{2}(L, L) \neq 0$, where $L$ is considered as a $K$-algebra. For example, we may take $K=(\mathbb{Z} / 2 \mathbb{Z})(x)$ with indeterminate $x$ and $L=K[y] /\left(y^{2}-x\right)$, and easily check that the algebra $L[y] /\left(y^{2}-x\right)^{2}$ is a non-splittable extension of the $K$-algebra $L$ over itself. Now take a 2-cocycle $\alpha: L \times L \rightarrow L$ corresponding to a non-splittable extension $0 \rightarrow L \rightarrow E \rightarrow L \rightarrow 0$. Let $Q=\left(Q_{0}, Q_{1}\right)$ be a finite quiver with the set of vertices $Q_{0}$ and the set of arrows $Q_{1}$. Assume $Q$ has no oriented cycles and double arrows. Denote by $Q^{+}$the set of all paths of length $\geq 1$ in $Q$. Consider the path algebra $H=L Q$ of $Q$ over $L$. Then $D(H) \simeq \operatorname{Hom}_{K}(L Q, K)$ for $D=\operatorname{Hom}_{L}(-, L)$. Corresponding to each vertex $i$ of $Q$, choose an idempotent $e_{i}$ of $H$ and corresponding to each arrow $\beta$ from $i$ to $j$ in $Q$ choose an element $h_{\beta}=e_{j} h_{\beta} e_{i}$ of $H$. For each path $p=\beta_{t} \ldots \beta_{1} \in Q^{+}$, we set $h_{p}=h_{\beta_{t}} \ldots h_{\beta_{1}}$. Then $D(H)$ has a basis $\left\{e_{i}^{*}, h_{p}^{*} \mid i \in Q_{0}, p \in Q^{+}\right\}$as an $L$-space. Let $\widetilde{H}=H \oplus D(H)$ be the direct sum of $K$-spaces and define multiplication on $\widetilde{H}$ in the following way:

$$
(a, u)(b, v)=\left(a b, a v+u b+\sum_{i \in Q_{0}} \alpha\left(a_{i}, b_{i}\right) e_{i}^{*}\right)
$$

for $a, b \in H, u, v \in D(H)$, where $a_{i}$ and $b_{i}$ are elements of $L$ such that

$$
a=\sum_{i \in Q_{0}} a_{i} e_{i}+\sum_{p \in Q^{+}} r_{p} h_{p} \quad \text { and } \quad b=\sum_{i \in Q_{0}} b_{i} e_{i}+\sum_{p \in Q^{+}} s_{p} h_{p}
$$

for $r_{p}, s_{p} \in L$. This multiplication with the usual addition of $K$-spaces makes $\widetilde{H}$ a $K$-algebra. It is shown in [26, Proposition 6.1] that the $K$-algebra extension

$$
0 \longrightarrow D(H) \longrightarrow \widetilde{H} \stackrel{\varrho}{\longrightarrow} H \longrightarrow 0
$$

with the canonical morphism $\varrho$ and embedding $D(H) \hookrightarrow \widetilde{H}$ is not splittable. Further, $\widetilde{H}$ is a selfinjective $K$-algebra [27], the elements $\widetilde{e}_{i}=\left(e_{i},-\alpha(1,1) e_{i}\right) \in$ $H \oplus D(H)=\widetilde{H}, i \in Q_{0}$, form a complete set of primitive orthogonal idempotents 
of $\widetilde{H}$, and $\left(1,-\alpha(1,1) \sum_{i \in Q_{0}} e_{i}^{*}\right)$ is the identity of $\widetilde{H}$. Clearly, the ordinary quiver of the $K$-algebra $H$ has no oriented cycles, $D(H)^{2}=0$ (in $\widetilde{H}$ ), and $D(H)$ is an injective cogenerator of $\bmod H$. Hence, by the above theorem $\widetilde{H}$ is socle equivalent to the trivial extension $H \ltimes D(H)$. On the other hand, $\widetilde{H}$ is not isomorphic to $H \ltimes D(H)$. We refer also to [28] for more information on nonsplittable extensions of algebras.

\section{Selfinjective Algebras WITH NON-PERIODIC AUsLandeR-REITEN COMPONENTS}

The aim of this section is to determine the structure of selfinjective artin algebras whose Auslander-Reiten quiver contains non-periodic generalized standard subquivers.

For an artin algebra $\Lambda$, we denote by $\Gamma_{\Lambda}$ the Auslander-Reiten quiver of $\Lambda$, and by $\tau_{\Lambda}$ and $\tau_{\Lambda}^{-}$the Auslander-Reiten translations $D \operatorname{Tr}$ and $\operatorname{Tr} D$, respectively. We shall identify the vertices of $\Gamma_{\Lambda}$ with the corresponding indecomposable $\Lambda$ modules. By a component of $\Gamma_{\Lambda}$ we mean a connected component of the quiver $\Gamma_{\Lambda}$. A component $\Gamma$ of $\Gamma_{\Lambda}$ is called regular if $\Gamma$ contains neither a projective nor an injective module. A subquiver $\Sigma$ of $\Gamma_{A}$ is said to be right stable (respectively, left stable) if $\tau_{\Lambda}^{-}$(respectively, $\tau_{\Lambda}$ ) is defined on all modules in $\Sigma$. A subquiver $\mathcal{C}$ is called non-periodic if $\mathcal{C}$ does not contain $\tau_{A}$-periodic modules, that is, modules $X$ with $X=\tau_{\Lambda}^{m} X$ for some $m \geq 1$. Following [24] a subquiver $\mathcal{D}$ of $\Gamma_{A}$ is said to be generalized standard if $\operatorname{rad}_{\Lambda}^{\infty}(X, Y)=0$ for all modules $X$ and $Y$ in $\mathcal{D}$. Recall that $\operatorname{rad}_{\Lambda}^{\infty}(X, Y)$ is the intersection of all finite powers $\operatorname{rad}^{m}(X, Y)$, for $m \geq 1$, of the radical $\operatorname{rad}(X, Y)$ of $\operatorname{Hom}_{\Lambda}(X, Y)$. Finally, the annihilator $r_{\Lambda}(\mathcal{E})$ of a subquiver $\mathcal{E}$ of $\Gamma_{\Lambda}$ in $\Lambda$ is the intersection of the (right) annihilators $r_{\Lambda}(X)$ of all modules $X$ in $\mathcal{E}$. Clearly, $r_{\Lambda}(\mathcal{E})$ is an ideal of $\Lambda$.

If $\Lambda$ is a selfinjective artin algebra, then $\tau_{\Lambda}$ and $\tau_{\Lambda}^{-}$are defined on all indecomposable $A$-modules except the projective-injective ones. Moreover, for each indecomposable projective-injective $\Lambda$-module $P$, we have an Auslander-Reiten sequence

$$
0 \rightarrow \operatorname{rad} P \rightarrow(\operatorname{rad} P / \operatorname{soc} P) \oplus P \rightarrow P / \operatorname{soc} P \rightarrow 0,
$$

and hence $\operatorname{rad} P$ is a unique direct predecessor of $P$ and $P / \operatorname{soc} P$ is a unique direct successor of $P$ in $\Gamma_{\Lambda}$. Hence, the Auslander-Reiten quiver $\Gamma_{\Lambda / \operatorname{soc} \Lambda}$ of $\Lambda / \operatorname{soc} \Lambda$ is obtained from $\Gamma_{\Lambda}$ by deleting the projective-injective modules $P$ and the arrows $\operatorname{rad} P \rightarrow P$ and $P \rightarrow P / \operatorname{soc} P$. For a component $\mathcal{C}$ of $\Gamma_{\Lambda}$, we denote by $\mathcal{C}^{\prime}$ the subquiver of $\Gamma_{\Lambda / \text { soc } \Lambda}$ obtained from $\mathcal{C}$ in this way. Obviously, if $\Lambda$ is representationinfinite, then $\mathcal{C}^{\prime}$ is a component of $\Gamma_{\Lambda / \text { soc } \Lambda}$. Observe also that if $\Gamma_{\Lambda}$ contains a nonperiodic right stable (respectively, left stable) subquiver, then $\Lambda$ is representationinfinite.

Proposition 5.1. Let $\Lambda$ and $A$ be two socle equivalent selfinjective artin algebras, and $\Phi: \bmod \Lambda / \operatorname{soc} \Lambda \rightarrow \bmod A / \operatorname{soc} A$ the isomorphism of module categories induced by an algebra isomorphism $\Lambda / \operatorname{soc} \Lambda \rightarrow A / \operatorname{soc} A$.

(i) Let $\Gamma$ be a right stable (respectively, left stable) full translation subquiver of $\Gamma_{\Lambda}$ which is closed under successors (respectively, predecessors) in $\Gamma_{\Lambda}$, and $\Sigma=\Phi(\Gamma)$. Then $\Gamma$ is generalized standard if and only if $\Sigma$ is generalized standard. 
(ii) Let $\mathcal{C}$ be a non-periodic component of $\Gamma_{\Lambda}$ and $\mathcal{D}$ a (non-periodic) component of $\Gamma_{A}$ such that $\Phi\left(\mathcal{C}^{\prime}\right)=\mathcal{D}^{\prime}$. Then $\mathcal{C}$ is generalized standard if and only if $\mathcal{D}$ is generalized standard.

Proof. (i) Since $\Gamma$ is right stable (respectively, left stable), it consists of $\Lambda / \operatorname{soc} \Lambda$ modules, and $\operatorname{soc} \Lambda \subseteq r_{\Lambda}(\Gamma)$. Applying now our second assumption that $\Gamma$ is closed under successors (respectively, predecessors) we deduce (see [23]) that $\Gamma$ is generalized standard as a subquiver of $\Gamma_{\Lambda}$ if and only if $\Gamma$ is generalized standard as a subquiver of $\Gamma_{\Lambda / \text { soc } \Lambda}$. Clearly, $\Sigma=\Phi(\Gamma)$ is also a right stable (respectively, left stable) full translation subquiver of $\Gamma_{A}$ which is closed under successors (respectively, predecessors) in $\Gamma_{A}$. Hence $\Sigma$ is generalized standard as a subquiver of $\Gamma_{A}$ if and only if $\Sigma$ is generalized standard as a subquiver of $\Gamma_{A / \operatorname{soc} A}$. Then the required equivalence follows.

(ii) Since $\mathcal{C}$ and $\mathcal{D}$ are non-periodic, it follows from [30] (see also [16]) that $\mathcal{C}$ and $\mathcal{D}$ have no oriented cycles. Then applying [17] we infer that $\mathcal{C}$ admits a left stable full translation subquiver $\Theta$ which is closed under predecessors and intersects the $\tau_{A^{-}}$-orbits of all nonprojective modules of $\mathcal{C}$. Dually, $\mathcal{C}$ admits a right stable full translation subquiver $\Omega$ which is closed under successors and intersects the $\tau_{A}^{-}$orbits of all nonprojective modules of $\mathcal{C}$. Then we may assume that $\Phi(\Theta)$ is a left stable full translation subquiver of $\mathcal{D}$ which is closed under predecessors and intersects the $\tau_{A}$-orbits of all nonprojective modules of $\mathcal{D}$, and $\Phi(\Omega)$ is a right stable full translation subquiver of $\mathcal{D}$ which is closed under successors and intersects the $\tau_{A}^{-}$-orbits of all nonprojective modules of $\mathcal{D}$. We know from [23] that $\mathcal{C}$ is generalized standard if and only if $\operatorname{rad}_{\Lambda}^{\infty}(X, Y)=0$ for all modules $X$ from $\Omega$ and $Y$ from $\Theta$. But $\Theta$ and $\Omega$ consist entirely of $\Lambda / \operatorname{soc} \Lambda$-modules. Hence, as above, we deduce that $\operatorname{rad}_{\Lambda}^{\infty}(X, Y)=\operatorname{rad}_{\Lambda / \mathrm{soc} \Lambda}^{\infty}(X, Y)$ for all modules $X$ from $\Omega$ and $Y$ from $\Theta$. Applying again [23], we obtain that $\mathcal{C}$ is a generalized standard component of $\Gamma_{A}$ if and only if $\mathcal{C}^{\prime}$ is a generalized standard component of $\Gamma_{\Lambda / \operatorname{soc} \Lambda}$. Similarly, we show that $\mathcal{D}$ is a generalized standard component of $\Gamma_{A}$ if and only if $\mathcal{D}^{\prime}$ is a generalized standard component of $\Gamma_{A / \operatorname{soc} A}$. This finishes the proof.

Now let $H$ be a basic and connected hereditary artin algebra over $K, \Delta$ the ordinary (valued) quiver of $H$, and $n$ the number of vertices in $\Delta$. Let $T$ be a multiplicity-free tilting $H$-module, that is, $\operatorname{Ext}_{H}^{1}(T, T)=0$ and $T$ is a direct sum of $n$ pairwise nonisomorphic indecomposable $H$-modules (see [4], [14]). Then $B=\operatorname{End}_{H}(T)$ is called a tilted algebra of type $\Delta$. The module $T$ determines a torsion theory $(\mathcal{F}(T), \mathcal{G}(T))$ in $\bmod H$, where $\mathcal{F}(T)=\left\{X \in \bmod H \mid \operatorname{Hom}_{H}(T, X)=\right.$ $0\}, \mathcal{G}(T)=\left\{X \in \bmod H \mid \operatorname{Ext}_{H}^{1}(T, H)=0\right\}$, and a splitting torsion theory $(\mathcal{Y}(T), \mathcal{X}(T))$ in $\bmod B$, where $\mathcal{Y}(T)=\left\{Z \in \bmod B \mid \operatorname{Tor}_{1}^{B}(Z, T)=0\right\}, \mathcal{X}(T)=$ $\left\{Z \in \bmod B \mid Z \otimes_{B} T=0\right\}$. By the Brenner-Butler theorem the functor $\operatorname{Hom}_{H}(T,-)$ induces an equivalence of $\mathcal{G}(T)$ and $\mathcal{Y}(T)$. The indecomposable direct summands of $\operatorname{Hom}_{H}(T, D(H))$ belong to one connected component $\mathcal{C}_{T}$ of $\Gamma_{B}$, called the connecting component of $\Gamma_{B}$ determined by $T$. This component connects the torsion-free part $\mathcal{Y}(T)$ of $\bmod B$ and the torsion part $\mathcal{X}(T)$ of $\bmod B$. The component $\mathcal{C}_{T}$ is generalized standard, because it does not contain oriented cycles and there are no nonzero maps from the torsion modules to the torsion-free modules. Moreover, $\mathcal{C}_{T}$ is regular if and only if $T$ is regular (a direct sum of indecomposable regular $H$ modules). It was shown in [21] that $H$ admits a regular tilting module if and only if $\Delta$ is neither a Dynkin nor a Euclidean (extended Dynkin) quiver and has more 
than two vertices. Consider now the repetitive algebra $\widehat{B}$ of $B$ and an infinite cyclic group $G$ acting freely on the objects and with finitely many orbits. Then $\Lambda=\widehat{B} / G$ is a selfinjective artin $K$-algebra and we have a Galois covering $F: \widehat{B} \rightarrow \Lambda$ with group $G$. Denote by $F_{\lambda}: \bmod \widehat{B} \rightarrow \bmod \Lambda$ the push-down functor induced by $F$ (see [5]). Assume that $\Delta$ is not a Dynkin quiver. Then, by [1], [22], [18] and [10], we know that

$$
\Gamma_{\widehat{B}}=\bigvee_{p \in \mathbb{Z}}\left(\mathcal{X}_{p} \vee \mathcal{R}_{p}\right)
$$

where, for each $p \in \mathbb{Z}, \mathcal{R}_{p}$ is a family of components whose stable parts are tubes (if $\Delta$ is Euclidean) or of type $\mathbb{Z} \mathbb{A}_{\infty}$ (if $\Delta$ is wild), and $\mathcal{X}_{p}$ is a component with the stable part of the form $\mathbb{Z} \Delta, \nu_{\widehat{B}}\left(\mathcal{R}_{p}\right)=\mathcal{R}_{p+2}$ and $\nu_{\widehat{B}}\left(\mathcal{X}_{p}\right)=\mathcal{X}_{p+2}$. Further, $\operatorname{Hom}_{\widehat{B}}\left(\mathcal{R}_{p}, \mathcal{X}_{p}\right)=0$ and $\operatorname{Hom}_{\widehat{B}}\left(\mathcal{R}_{p} \vee \mathcal{X}_{p}, \mathcal{R}_{q} \vee \mathcal{X}_{q}\right)=0$ for $p, q \in \mathbb{Z}, p<q$. Since $G$, considered as group of automorphisms of $\widehat{B}$, acts freely on the indecomposable projective $\widehat{B}$-modules, it also acts freely on the components of $\Gamma_{\widehat{B}}$. Moreover, we know that $\widehat{B}$ is locally-support finite [8], that is, for each object $x$ of $\widehat{B}$ the full subcategory of $\widehat{B}$ consisting of the supports of indecomposable finitely generated $\widehat{B}$-modules having $x$ in its support has finitely many objects. Applying [7] (see also [8]) and [12] we conclude that the push-down functor $F_{\lambda}: \bmod \widehat{B} \rightarrow \bmod \Lambda$ is dense and preserves the Auslander-Reiten sequences. Therefore, $\Gamma_{\Lambda}$ is obtained from $\Gamma_{\widehat{B}}$ by identifying (via $\left.F_{\lambda}\right) \mathcal{X}_{p}$ with $\mathcal{X}_{p+m}$ and $\mathcal{R}_{p}$ with $\mathcal{R}_{p+m}$, for some $m \geq 1$ and all $p \in \mathbb{Z}$. Thus $\Gamma_{\Lambda}$ is of the form

$$
F_{\lambda}\left(\mathcal{X}_{0} \vee \mathcal{R}_{0}\right) \vee F_{\lambda}\left(\mathcal{X}_{1} \vee \mathcal{R}_{1}\right) \vee \ldots \vee F_{\lambda}\left(\mathcal{X}_{m-1} \vee \mathcal{R}_{m-1}\right)
$$

We have also the following facts on $\widehat{B}$ and $\Lambda$.

Proposition 5.2. There are two tilted algebras $B_{1}=\operatorname{End}_{H}\left(T_{1}\right), B_{2}=\operatorname{End}_{H}\left(T_{2}\right)$, where $T_{1}$ is a tilting $H$-module without nonzero preprojective direct summands and $\mathrm{T}_{2}$ is a tilting $\mathrm{H}$-module without nonzero preinjective direct summands, such that $\widehat{B}_{1} \simeq \widehat{B} \simeq \widehat{B}_{2}$.

Proof. See [1], for $\Delta$ Euclidean, and [18], for $\Delta$ wild.

Proposition 5.3. The following conditions are equivalent:

(i) $G$ is generated by an element $\varphi \nu_{\widehat{B}}$, where $\varphi$ is a positive automorphism of $\widehat{B}$.

(ii) There exists $p, 0 \leq p \leq m-1$, such that $F_{\lambda}\left(\mathcal{X}_{p}\right)$ contains a generalized standard right stable full translation subquiver $\Sigma_{p}$ which is closed under successors.

(iii) There exists $q, 0 \leq q \leq m-1$, such that $F_{\lambda}\left(\mathcal{X}_{q}\right)$ contains a generalized standard left stable full translation subquiver $\Theta_{q}$ which is closed under predecessors.

(iv) For each $i, 0 \leq i \leq m-1, F_{\lambda}\left(\mathcal{X}_{i}\right)$ contains a generalized standard right stable full translation subquiver $\Sigma_{i}$ which is closed under successors and a generalized standard left stable full translation subquiver $\Theta_{i}$ which is closed under predecessors.

Proof. See [1], [18], if $\Delta$ is Euclidean, and [10] if $\Delta$ is wild.

Proposition 5.4. Assume that $F_{\lambda}\left(\mathcal{X}_{p}\right)$, for some $0 \leq p \leq m-1$, is nonregular. Then the following conditions are equivalent: 
(i) $G$ is generated by an element $\varphi \nu_{\widehat{B}}$, where $\varphi$ is a strictly positive automorphism of $\widehat{B}$.

(ii) The component $F_{\lambda}\left(\mathcal{X}_{p}\right)$ is generalized standard.

(iii) All components $F_{\lambda}\left(\mathcal{X}_{q}\right), 0 \leq q \leq m-1$, are generalized standard.

Proof. See [1], [22], if $\Delta$ is Euclidean, and [10] if $\Delta$ is wild.

We are now able to prove our main results on the structure of selfinjective artin algebras whose Auslander-Reiten quiver contains a non-periodic generalized standard right (left) stable subquiver. We assume that $A$ is a basic and connected selfinjective artin algebra over a commutative artin ring $K$.

Theorem 5.5. The following conditions are equivalent:

(i) $\Gamma_{A}$ admits a non-periodic generalized standard right stable full translation subquiver which is closed under successors in $\Gamma_{A}$.

(ii) $A$ is socle equivalent to $\widehat{B} /\left(\varphi \nu_{\widehat{B}}\right)$, where $B$ is a tilted algebra of the form $\operatorname{End}_{H}(T)$, for some hereditary artin algebra $H$ and a tilting $H$-module $T$ without nonzero preprojective direct summands, and $\varphi$ is a positive automorphism of $\widehat{B}$.

(iii) $A$ is socle equivalent to $\widehat{B} /\left(\varphi \nu_{\widehat{B}}\right)$, where $B$ is a tilted artin $K$-algebra not of Dynkin type and $\varphi$ is a positive automorphism of $\widehat{B}$.

(iv) $A$ is socle equivalent to $\widehat{B} /\left(\varphi \nu_{\widehat{B}}\right)$, where $B$ is a tilted algebra of the form End $_{H}(T)$, for some hereditary artin $K$-algebra $H$ and a tilting $H$-module $T$ without nonzero preinjective direct summands, and $\varphi$ is a positive automorphism of $\widehat{B}$.

(v) $\Gamma_{A}$ admits a non-periodic generalized standard left stable full translation subquiver which is closed under predecessors in $\Gamma_{A}$.

Moreover, if $K$ is an algebraically closed field, we may replace in the above equivalences "socle equivalent" by "isomorphic".

Proof. The equivalence of (ii), (iii) and (iv) is a direct consequence of Proposition 5.2. Moreover, the implications (ii) $\Rightarrow$ (i) and (iv) $\Rightarrow(\mathrm{v})$ are consequences of Propositions 5.1 and 5.3. We shall prove that (i) implies (ii). The proof that (v) implies (iv) is similar.

Assume now that $\Gamma_{A}$ admits a non-periodic generalized standard right stable full translation subquiver $\Gamma$ which is closed under successors in $\Gamma_{A}$. Since $A$ is selfinjective, we then conclude that $\Gamma$ has no projective modules and oriented cycles. Applying [17] we get that $\Gamma$ contains a full translation subquiver $\mathcal{D}$ of the form $(-\mathbb{N}) \Delta$, for some valued quiver $\Delta$ without oriented cycles, which is closed under successors in $\Gamma_{A}$. Since $\mathcal{D}$ is also generalized standard, it follows from [25, Lemma 2] that $\Delta$ is finite. Let $I=r_{A}(\mathcal{D})$ be the annihilator of $\mathcal{D}$ in $A, B=A / I$ and $e$ a residual identity of $B$. We proved in [26, Theorem 5.1 and Proposition 5.3] that $I e I=0, I e$ is an injective cogenerator in $\bmod B$, and $B$ is a tilted algebra, having a complete slice of type $\Delta$ (in the sense of [[20], (4.2)]) formed by modules from $\mathcal{D}$. Hence, $B=\operatorname{End}_{H}(T)$, for a hereditary artin $K$-algebra $H$ of type $\Delta$ and a tilting $H$-module $T$. Moreover, $\mathcal{D}$ is a full translation subquiver of the connecting component $\mathcal{C}_{T}$ of $\Gamma_{B}$ which is closed under successors in $\Gamma_{B}$. Consequently, $T$ has no nonzero preprojective direct summands. Obviously, the ordinary quiver $Q_{B}$ of $B$ has no oriented cycles [20]. Applying now Theorem 4.1 we conclude that $A$ is socle equivalent to $\widehat{B} /\left(\varphi \nu_{\widehat{B}}\right)$, for a positive automorphism $\varphi$ of $\widehat{B}$. Hence, (i) implies (ii). 
If $K$ is an algebraically closed field, then the required equivalences follow from the above proof and the second part of Theorem 4.1.

Corollary 5.6. (i) $\Gamma_{A}$ admits a non-periodic nonregular generalized standard component if and only if $A$ is a socle equivalent to $\widehat{B} /\left(\varphi \nu_{\widehat{B}}\right)$, where $B$ is a tilted algebra of the form $B=\operatorname{End}_{H}(T)$, for some hereditary artin $K$-algebra $H$ and a nonregular tilting $H$-module $T$ without nonzero preprojective (respectively, preinjective) direct summands, and $\varphi$ is a strictly positive automorphism of $\widehat{B}$.

(ii) $\Gamma_{A}$ admits a non-periodic regular generalized standard component if and only if $A$ is socle equivalent to $\widehat{B} /\left(\varphi \nu_{\widehat{B}}\right)$, where $B$ is a tilted algebra of the form $\operatorname{End}_{H}(T)$, for some hereditary artin $K$-algebra $H$ and a regular tilting $H$-module $T$, and $\varphi$ is a positive automorphism of $\widehat{B}$.

Moreover, if $K$ is an algebraically closed field, we may replace in the above equivalences "socle equivalent" by "isomorphic".

Proof. It is a direct consequence of Theorem 5.5 and Propositions 5.1, 5.3 and 5.4.

Recall that an artin algebra $\Lambda$ is called symmetric if $\Lambda$ and $D(\Lambda)$ are isomorphic as $\Lambda$-bimodules, or equivalently the Nakayama automorphism of $\Lambda$ is the identity. It is well-known that the trivial extension $B \ltimes D(B)$ of any algebra $B$ by its injective cogenerator $D(B)$ is a symmetric algebra. We get also the following consequences of our main results and definition of $G$ in the proof of Theorem 3.1.

Corollary 5.7. Assume $A$ is symmetric. Then $\Gamma_{A}$ admits a non-periodic right stable (respectively, left stable) generalized standard full translation subquiver which is closed under successors (respectively, predecessors) in $\Gamma_{A}$ if and only if $A$ is socle equivalent to $B \ltimes D(B)$, where $B$ is a tilted $K$-algebra not of Dynkin type. Moreover, if $K$ is an algebraically closed field, we may replace "socle equivalent" by "isomorphic".

Corollary 5.8. Assume $A$ is symmetric. Then the following conditions are equivalent:

(i) $\Gamma_{A}$ admits a non-periodic generalized standard component.

(ii) $\Gamma_{A}$ admits a non-periodic regular generalized standard component.

(iii) $A$ is socle equivalent to $B \ltimes D(B)$, where $B$ is a tilted algebra of the form $\operatorname{End}_{H}(T)$, for a hereditary artin $K$-algebra $H$ and a regular tilting $H$-module $T$.

Moreover, if $K$ is an algebraically closed field, we may replace "socle equivalent" by "isomorphic".

\section{REFERENCES}

1. I. Assem, J. Nehring and A. Skowroński, Domestic trivial extensions of simply connected algebras, Tsukuba J. Math. 13 (1989), 31-72. MR 90j:16043

2. I. Assem and A. Skowroński, On tame repetitive algebras, Fund. Math. 142 (1993), 59-84. MR 94a:16022

3. M. Auslander, I. Reiten and S. O. Smalø, Representation Theory of Artin Algebras, Cambridge Studies in Adv. Math. 36 (Cambridge Univeristy Press, 1995). MR 96c:16015

4. K. Bongartz, Tilted algebras, in: Representations of Algebras, Lecture Notes in Math. 903, 26-38. MR 83g:16053

5. K. Bongartz and P. Gabriel, Covering spaces in representation theory, Inventiones Math. 65 (1981), 331-378. MR 84i:16030 
6. O. Bretscher, C. Läser and C. Riedtmann, Self-injective and simply connected algebras, Manuscripta Math. 36 (1981), 253-307. MR 84i:16021

7. P. Dowbor, H. Lenzing and A. Skowroński, Galois coverings of algebras by locally supportfinite categories, in: Representation Theory I. Finite Dimensional Algebras, Lecture Notes in Math. 1177, 91-93. MR 87j:16012

8. P. Dowbor and A. Skowroński, Galois coverings of representation-infinite algebras, Commentarii Math. Helvetici 62 (1987), 311-337. MR 88m:16020

9. K. Erdmann, Blocks of tame representation type and related algebras, Lecture Notes in Math. 1428. MR 91c: 20016

10. K. Erdmann, O. Kerner and A. Skowroński, Self-injective algebras of wild tilted type, J. Pure Appl. Algebra, in press.

11. K. Erdmann and A. Skowroński, On Auslander-Reiten components of blocks and biserial algebras, Transactions Amer. Math. Soc. 330 (1992), 165-189. MR 93b:16022

12. P. Gabriel, The universal cover of a representation-finite algebra, in: Representations of Algebras, Lecture Notes in Math. 903, 68-105. MR 83f:16036

13. D. Happel, On the derived category of a finite-dimensional algebra, Commentari Math. Helvetici 62 (1987), 339-389. MR 89c:16029

14. D. Happel and C. M. Ringel, Tilted algebras, Transactions Amer. Math. Soc. 274 (1982), 399-443. MR 84d:16027

15. D. Hughes and J. Waschbüsch, Trivial extensions of tilted algebras, Proc. London Math. Soc. 46 (1983), 347-364. MR 84m:16023

16. S. Liu, Degrees of irreducible maps and the shapes of the Auslander-Reiten quivers, J. London Math. Soc. 45 (1992), 32-54. MR 93f: 16015

17. S. Liu, Semi-stable components of an Auslander-Reiten quiver, J. London Math. Soc. 47 (1993), 405-416. MR 94a:16024

18. L. Peng and J. Xiao, Invariability of repetitive algebras of tilted algebras under stable equivalence, J. Algebra 170 (1994), 54-68. MR 95k:16023

19. C. Riedtmann, Representation-finite self-injective algebras of class $\mathbb{D}_{n}$, Compositio Math. 49 (1983), 231-282. MR 85e:16049

20. C. M. Ringel, Tame algebras and integral quadratic forms, Lecture Notes in Math. 1099. MR 87f: 16027

21. C. M. Ringel, The regular components of the Auslander-Reiten quiver of a tilted algebra, Chinese Ann. Math. 9B (1988), 1-18. MR 89e:16036

22. A. Skowroński, Selfinjective algebras of polynomial growth, Math. Annalen 285 (1989), 177199. MR 90k: 16024

23. A. Skowroński, Generalized standard Auslander-Reiten components without oriented cycles, Osaka J. Math. 30 (1993), 515-527. MR 94k:16026

24. A. Skowroński, Generalized standard Auslander-Reiten components, J. Math. Soc. Japan 46 (1994), 517-543. MR 95d:16022

25. A. Skowroński, Regular Auslander-Reiten components containing directing modules, Proc. Amer. Math. Soc. 120 (1994), 19-26. MR 94b:16021

26. A. Skowroński and K. Yamagata, Socle deformations of self-injective algebras, Proc. London Math. Soc. 72 (1996), 545-566. MR 96m:16004

27. K. Yamagata, Extensions over hereditary Artinian rings with self-dualities I, J. Algebra $\mathbf{7 3}$ (1981), 386-433. MR 83g:16030

28. K. Yamagata, Representations of nonsplittable extension algebras, J. Algebra 115 (1988), 32-45. MR 89d:16029

29. K. Yamagata, Frobenius Algebras, in: Handbook of Algebra, vol. 1, (North-Holland, 1996), pp. 841-887. MR 97k:16022

30. Y. Zhang, The structure of stable components, Canadian J. Math. 43 (1991), 652-672. MR 92f: 16017

FaCUlty of Mathematics and Informatics, Nicholas Copernicus University, ChopiNA 12/18, 87-100 TORuń, Poland

E-mail address: skowron@mat.uni.torun.pl

Department of Mathematics, Tokyo University of Agriculture and Technology, FuChU, TOKYO 183, JAPAN

E-mail address: yamagata@cc.tuat.ac.jp 